\title{
The phonon-coupling model for Skyrme forces
}

\author{
N. Lyutorovich and V. Tselyaev \\ V. A. Fock Institute of Physics, St. Petersburg State University, RU-198504 St. Petersburg, Russia \\ J. Speth* and S. Krewald \\ Institut für Kernphysik, Forschungszentrum Jülich, D-52425 Jülich, Germany \\ P.-G. Reinhard \\ Institut für Theoretische Physik II, Universität Erlangen-Nürnberg, D-91058 Erlangen, Germany
}

(Dated: June 19, 2018)

\begin{abstract}
A short review on the self-consistent RPA with in a energy-density functional of the Skyrme type is given. We also present an extension of the RPA where the coupling of phonons to the single particle states is considered. Within this approach we present numerical results which are compared with data. The self-consistent approach is compared with the Landau-Migdal theory. Here we derive from the self-consistent $p h$ interaction, the Landau-Migdal parameters as well as their density dependence. In the appendix a new derivation of the reduced $p h$ matrix elements is presented.

PACS numbers: 21.30.Fe,21.60.-n,21.60.Jz,24.30.Cz,21.10.-k
\end{abstract}

\section{INTRODUCTION}

In the present contribution we review the self consistent time blocking approximation (TBA) and present results obtained within this method. The conventional random phase approximation (RPA) allows, e.g., the calculation of the total transition strength and the mean energy of giant resonances. If one is interested in the distribution of the strength and cross sections of various nuclear reactions like electron scattering and $\alpha$ scattering it is necessary to consider in addition to the one-particle-one-hole $(1 \mathrm{p} 1 \mathrm{~h})$ states more complex configurations, e.g., two-particle-two-hole states [1] or the fragmentation of the single particle strength due to the coupling to phonons [2 [5]. As input data for those approaches one needs single particle energies and wave functions and a residual particle-hole interaction. In earlier works one started with a single particle model and defined a residual particle-hole interaction where both quantities where adjusted to experimental data [6]. The next stage of sophistication are self-consistent calculations where one starts with an effective Hamiltonian or a density functional from which one obtains by functional derivations the single particle quantities as well as the residual interaction [7, 8]. The phenomenology enters here in the Hamiltonian and the density functional in which remain free parameters which have to be adjusted to experimental data 9 -12.

In the formal part we present the self-consistent RPA equations within an energy-density functional approach and give a short derivation of the TBA. The selfconsistent approach is compared with the Landau-Migdal theory. Here we connect the "Landau-Migdal interaction" with the self-consistent $p h$-interaction and calculate

\footnotetext{
*J.Speth@fz-juelich.de
}

the famous Landau-Migdal parameters as well as their density dependence.

In all our calculations we started from a density functional of the Skyrme type where we used various sets of parameters. We found from our investigations that the inclusion of phonons give very good results for ${ }^{208} \mathrm{~Pb}$ and fair results for the Ca-isotopes for the giant isovector dipole resonance (GDR) as well as for the isoscalar resonances for some of the Skyrme parametrizations. A parameter set which reproduces all these properties simultaneously is still missing. In the light nucleus ${ }^{16} \mathrm{O}$ the GDR is well reproduces but not the isoscalar resonances. This will be discussed later.

An important point in the calculation of giant resonances is the treatment of the single particle continuum. In earlier calculations the continuum was discretized which introduced some arbitrariness. Most recently we developed a new method for handling the continuum that allows to consider also the spin-orbit interaction. Therefore we can now present results of the selfconsistent calculations which include also this part of the residual interaction.

In Chapter II we introduce the basic formulas of the self-consistent RPA and TBA. In Chapter III.A we introduce several different Skyrme parametrization and discuss their connections to nuclear matter properties. In Chapter III.B we compare the residual interaction of the Migdal type with the one which follows from the second derivative of the Skyrme type density functional. We derive from the residual Skyrme interaction the spin independent Migdal parameters and their density behavior. Finally we present in Chapter IV a large body of data which are compared with experimental data and in Chapter V we give a summary.

In the appendix we discuss in the subsection 1-4 in some detail the Skyrme energy-density functional in general and give detailed formulas for the Skyrme interac- 
tion. In subsection 5-9 we derive the reduced matrix elements of the residual $p h$-interaction which include not only the zero-range and velocity dependent parts but also the spin-orbit matrix elements.

\section{THE METHOD}

\section{A. Formulation of the Hamiltonian}

RPA is, in principle, a very general method in the context of many-body theory which emerges from collecting a subset of leading diagrams (bubble series) within the Green function formalism, for details and explicit expressions see [13. We will discuss here RPA in the context of effective interactions, or effective energy-density functionals respectively (for the expressions in a nuclear context see [14, 15]). This will result in somewhat different notations and aspects. Thus we will introduce here the basic ingredients as we will employ them in the sequel.

In many-body theory the one-particle Green function is determined by the mass operator $\Sigma$ which incorporates all information on the many-body system. Its most general form is

$$
\Sigma=\Sigma(\mathbf{r}, \mathbf{p}, \epsilon)
$$

depending on the coordinate $\mathbf{r}$, the momentum $\mathbf{p}$ (nonlocality) and the single-particle (s.p.) energy $\epsilon$ (dependence on the spin and isospin variables is tacitly implied). This $\Sigma$ is a highly non-local one-body operator. The first non-local feature is the effective mass which gathers contributions from $\mathbf{p}$-dependence as well as $\epsilon$-dependence [16. RPA is a theory for the response function $R$ which is a propagator for a coherent superposition of $1 p 1 h$ states. It involves, besides $\Sigma$, a residual two-body interaction which is derived in the many-body framework as

$$
K=\frac{\delta \Sigma}{\delta G}
$$

where $\delta$ stands for functional derivative and $G$ for the one-body Greens operator. Similar as $\Sigma, K$ is a highly non-local and energy-dependent operators, now even more involved because it acts on two particles.

Effective interactions as the Landau-Migdal interaction [17, 18, or the Skyrme force [19 21] are free from energy dependence, but may carry some momentum dependence of second order in $\mathbf{p}$, for detailed discussion see section IIIA. In these cases the equations-of-motion become much simpler, e.g., as the single-particle strength is simply one throughout [22. This is also indicated by using slightly different notations. The place of $\Sigma$-operator is now taken by the mean-field Hamiltonian

$$
\hat{h} \equiv h_{12}
$$

which is a one-body operator at most of order $\mathbf{p}^{2}$. The numerical indices here and in the following denote the set of the quantum numbers of some single-particle basis. In the context of the density functional theory (DFT) with the energy density functional $E[\rho]$, the mean-field Hamiltonian is deduced from $E[\rho]$ by functional derivative

$$
h_{12}=\frac{\delta E}{\delta \rho_{21}} .
$$

In practice, RPA described excitation about the stationary ground state and we deal with the ground-state mean-field Hamiltonian that is simultaneously diagonal with $\rho$. It is convenient to write it in the basis which diagonalizes operators $h$ and $\rho$, yielding

$$
h_{12}=\varepsilon_{1} \delta_{12}, \quad \rho_{12}=n_{1} \delta_{12},
$$

where $n_{1}=0,1$ is the occupation number. In what follows the indices $p$ and $h$ will be used to label the single-particle states of the particles $\left(n_{p}=0\right)$ and holes $\left(n_{h}=1\right)$ in this basis.

The residual two-body interaction for RPA is derived again from eq. $1 \mathrm{~b}$, now replacing $\Sigma$ by $\hat{h}$ and $G$ by $\hat{\rho}$. This yields for the case of DFT eventually

$$
V_{12,34}=\frac{\delta^{2} E[\rho]}{\delta \rho_{21} \delta \rho_{34}},
$$

so the quantities $h$ and $V$ appear to be linked by Eqs. (2b) and (3). Other effective interactions, as the LandauMigdal interaction, are modeled directly as residual twobody interaction at the level of $K$.

\section{B. Self-consistent RPA}

Our approach is based on the version of the response function formalism developed within the Green function method (see Ref. [13). The aim is to compute a nuclear transition strength for an observable corresponding to some one-body operator $Q$. The strength function $S_{Q}(E)$ is defined in terms of the response function $R(\omega)$ by

$$
\begin{aligned}
& S(E)=-\frac{1}{\pi} \operatorname{Im} \Pi(E+i \Delta), \\
& \Pi(\omega)=-\langle Q|R(\omega)| Q\rangle,
\end{aligned}
$$

where $E$ is the excitation energy, $\Delta$ a smearing parameter simulating broadening effects beyond RPA, and $\Pi(\omega)$ the (dynamic) $Q$-polarizability.

The strength functions combines system properties with an observable. The system property is the response function $R$ which at RPA level is a solution of the BetheSalpeter equation (BSE)

$$
R^{\mathrm{RPA}}(\omega)=R^{(0)}(\omega)-R^{(0)}(\omega) V R^{\mathrm{RPA}}(\omega),
$$

where $R^{(0)}(\omega)$ is the uncorrelated $1 p 1 h$ propagator and $V$ is the residual two-body interaction (see section II A). The $1 p 1 h$ propagator $R^{(0)}(\omega)$ is defined as

$$
R^{(0)}(\omega)=-\left(\omega-\Omega^{(0)}\right)^{-1} M^{\mathrm{RPA}},
$$


where the matrices $\Omega^{(0)}$ and $M^{\mathrm{RPA}}$ are defined in the $1 p 1 h$ configuration space. $M^{\mathrm{RPA}}$ is the metric matrix defined (in the diagonal basis) as

$$
M_{12,34}^{\mathrm{RPA}}=\delta_{13} \delta_{24}\left[n_{2}-n_{1}\right] .
$$

The matrix $\Omega^{(0)}$ comprises the one-body Hamiltonian acting separately on particle and hole in the form

$$
\Omega_{12,34}^{(0)}=\delta_{13} \delta_{24}\left[\varepsilon_{1}-\varepsilon_{2}\right] .
$$

The propagator $R^{\mathrm{RPA}}(\omega)$, being a matrix in $1 p 1 h$ space, is a rather bulky object. For practical calculations, it is more convenient to express it in terms of RPA amplitudes $z_{12}^{n}$ by virtue of the spectral representation

$$
R_{1234}^{\mathrm{RPA}}(\omega)=-\sum_{n} z_{12}^{n} \frac{\operatorname{sgn}\left(\omega_{n}\right)}{\omega-\omega_{n}}\left(z_{34}^{n}\right)^{*}
$$

where $n$ labels the RPA eigenmodes and $\omega_{n}$ is the eigenfrequency. Inserting that into Eq. (5) and filtering the pole at $\omega=\omega_{n}$ yields the familiar RPA equations

$$
\sum_{34}\left(\Omega_{12,34}^{(0)}+\sum_{56} M_{12,56}^{\mathrm{RPA}} V_{56,34}\right) z_{34}^{n}=\omega_{n} z_{12}^{n},
$$

where the transition amplitudes $z^{n}$ are normalized by the condition

$$
\sum_{12,34}\left(z_{12}^{n}\right)^{*} M_{1234}^{\mathrm{RPA}} z_{34}^{n^{\prime}}=\operatorname{sgn}\left(\omega_{n}\right) \delta_{n, n^{\prime}} .
$$

These equations determine the set of eigenstates $n$ with amplitudes $z_{12}^{n}$ and frequencies $\omega_{n}$.

\section{Phonon coupling model}

The second model is the quasiparticle-phonon coupling model within the time-blocking approximation (TBA) [4, 6, 23, 24] (without ground state correlations beyond the RPA included in [4, 6, 23, 24] and without pairing correlations included in 24]). This model, which in the following will be referred to as the TBA, is an extension of the RPA including $1 p 1 h \otimes$ phonon configurations in addition to the $1 p 1 h$ configurations incorporated in the conventional RPA. The BSE for the response function in the TBA is

$$
\begin{aligned}
R^{\mathrm{TBA}}(\omega)= & R^{(0)}(\omega) \\
& -R^{(0)}(\omega)(V+\tilde{W}(\omega)) R^{\mathrm{TBA}}(\omega), \\
\tilde{W}(\omega)= & W(\omega)-W(0),
\end{aligned}
$$

where the induced interaction $\tilde{W}(\omega)$ serves to include contributions of $1 p 1 h \otimes$ phonon configurations. The matrix $W(\omega)$ in Eq. (13) is defined in the $1 p 1 h$ subspace and can be represented in the form

$$
W_{12,34}(\omega)=\sum_{c, \sigma} \frac{\sigma F_{12}^{c(\sigma)} F_{34}^{c(\sigma) *}}{\omega-\sigma \Omega_{c}},
$$

where $\sigma= \pm 1, c=\left\{p^{\prime}, h^{\prime}, n\right\}$ is an index of the subspace of $1 p 1 h \otimes$ phonon configurations, $n$ is the phonon's index,

$$
\begin{gathered}
\Omega_{c}=\varepsilon_{p^{\prime}}-\varepsilon_{h^{\prime}}+\omega_{n}, \quad \omega_{n}>0, \\
F_{12}^{c(-)}=F_{21}^{c(+) *}, \quad F_{p h}^{c(-)}=F_{h p}^{c(+)}=0, \\
F_{p h}^{c(+)}=\delta_{p p^{\prime}} g_{h^{\prime} h}^{n}-\delta_{h^{\prime} h} g_{p p^{\prime}}^{n},
\end{gathered}
$$

and $g_{12}^{n}$ is an amplitude of the quasiparticle-phonon interaction. These $g$ amplitudes (along with the phonon's energies $\omega_{n}$ ) are determined by the positive frequency solutions of the RPA equations and the emerging $z$ amplitudes as

$$
g_{12}^{n}=\sum_{34} V_{12,34} z_{34}^{n}
$$

In our DFT-based approach the energy density functional $E[\rho]$ in Eqs. (2b) and (3) is the functional of the Skyrme type with model parameters adjusted to reproduce nuclear ground state properties with high quality. In this case $E[\rho]$ already effectively contains a part of the contributions of those $1 p 1 h \otimes$ phonon configurations which are explicitly included in the TBA. Therefore, in a theory going beyond the RPA, the problem of double counting arises. To avoid this problem in the TBA, we use the subtraction method. It consists in the replacement of the amplitude $W(\omega)$ by the quantity $\tilde{W}(\omega)=W(\omega)-W(0)$ as it is written in Eq. 12). In Ref. 25] it was shown that, in addition to the elimination of double counting, the subtraction method ensures stability of solutions of the TBA eigenvalue equations.

\section{EFFECTIVE INTERACTIONS}

\section{A. Basics on the Skyrme functional and related parameters}

From the variety of self-consistent nuclear mean-field models 10, we consider here a non-relativistic branch, the widely used and very successful Skyrme-Hartree-Fock (SHF) functional. A detailed description of the functional is given in the appendix and more background information can be found in the reviews 10, 26, 27. We summarize here the essential features. The functional depends on a couple of local densities and currents (density, gradient of density, kinetic-energy density, spin-orbit density, current, spin density, kinetic spindensity). For the description of ground state properties and natural-parity excitations, there remain typically 13 14 free model parameters. They are usually determined by a fit to a large and representative set of experimental data on bulk properties of the nuclear ground state, for recent adjustments see [11, 28, 29]. The parameters thus found are considered to be universal parameters as they apply to all nuclei throughout the nuclear landscape and to astro-physical matter (e.g. neutron stars). 


\begin{tabular}{l|cccc} 
& $K[\mathrm{MeV}]$ & $m^{*} / m$ & $a_{\text {sym }}[\mathrm{MeV}]$ & $\kappa_{\mathrm{TRK}}$ \\
\hline SV-bas & 234 & 0.90 & 30 & 0.4 \\
SV-kap00 & 234 & 0.90 & 30 & 0.0 \\
SV-mas07 & 234 & 0.70 & 30 & 0.4 \\
SV-sym34 & 234 & 0.90 & 34 & 0.4 \\
SV-K218 & 218 & 0.90 & 30 & 0.4 \\
SV-m64k6 & 241 & 0.64 & 27 & 0.6 \\
SV-m56k6 & 255 & 0.56 & 27 & 0.6 \\
\hline
\end{tabular}

TABLE I. NMP for the Skyrme paramterizations used in this study: incompressibility $K$, isoscalar effective mass $m^{*} / m$, symmetry energy $a_{\text {sym }}$, Thomas-Reiche-Kuhn sum rule enhancement $\kappa_{\mathrm{TRK}}$. The first five parametrizations stem from [28], the last two from [7]. For the definition of the NMP, see appendix A.

The force parameters $C_{T}^{(\text {typ })}$, although necessary for communicating the model and coding, are not very intuitive. The most important part of the functional can be characterized by nuclear matter properties (NMP), i.e. equilibrium properties of homogeneous, symmetric nuclear matter, for which we have some intuition from the liquid-drop model (LDM) 30. A detailed definition of the NMP is given in appendix A4. Of particular interest for resonance excitations are the NMP which are related to response to perturbations: incompressibility $K$ (isoscalar static), effective mass $m^{*} / m$ (isoscalar dynamic), symmetry energy $a_{\text {sym }}$ (isovector static), TRK sum rule enhancement $\kappa_{\mathrm{TRK}}$ (isovector dynamic). It turns out that a fit to ground state properties fixes some of the NMP very well (equilibrium density and binding energy, to some extend also incompressibility) while others are left with an appreciable leeway (particularly the isovector properties symmetry energy and sum-rule enhancement). This calls for careful evaluation of the predictive value of SHF calculations. There is a great manifold of strategies to explore the uncertainties in predictions, for recent discussion see, e.g., 3133.

One such strategy is a systematic variation of properties of a functional in the vicinity of the optimal fit. And it is obvious that one should vary the most important agents which are for the SHF functional the NMP. In that spirit, the paper 28] provides a series SHF parametrizations with systematically varied NMP. We use these sets here to explore the sensitivity, or robustness, of the phonon coupling under variations of the functional in reasonable ranges (i.e. in the vicinity of the optimum). Table I lists the selection of parametrizations and their NMP. SV-bas is the base point of the variation of forces. Its NMP are chosen such that dipole polarizability and the three most important giant resonances (GMR, GDR, and GQR) in ${ }^{208} \mathrm{~Pb}$ are well reproduced by Skyrme-RPA calculations. Each one of the next four parametrizations vary exactly one NMP while keeping the other three at the SV-bas value. These $1+4$ parametrizations allow to explore the effect of each NMP separately. It was figured out in 28] that there is a unique relation between each one of the four NMP and one resonance in ${ }^{208} \mathrm{~Pb}: K$ affects mainly the GMR, $m^{*} / m$ af- fects mainly the GQR, $\kappa_{\mathrm{TRK}}$ affects mainly the GDR, and $a_{\text {sym }}$ is uniquely linked to the dipole polarizability [34]. Finally, the last two parametrizations in table $\mathrm{I}$ were developed in [7] with the goal to describe, within TBA, at the same time the GDR in ${ }^{16} \mathrm{O}$ and ${ }^{208} \mathrm{~Pb}$. This required to push up the RPA peak energy which was achieved by low $a_{\text {sym }}$ in combination with high $\kappa_{\text {TRK }}$. To avoid unphysical spectral distributions for the GDR, a very low $m^{*} / m$ was used.

\section{B. From Landau-Migdal theory to the SHF residual interaction}

The SHF theory provides reliable nuclear ground states and with it the first ingredients for the RPA/TBA equations, namely s.p. wavefunctions, mean-field Hamiltonian $\hat{h}$ and corresponding s.p. energies $\varepsilon_{i}$. The crucial piece, going beyond ground state properties, is the effective residual interaction. It can be derived fully selfconsistency from the SHF functional with Eq. (3). The details of its evaluation for spherical nuclei are given in appendix A5-A9. In these sections, we will briefly review the development of effective residual interactions which started out with the Landau-Migdal interaction in the context of Fermi liquid theory [17] and compare it with the actual form delivered by the SHF functional, for a more extensive discussion see 35 .

The theory of Fermi liquids deals with homogeneous matter where momentum space provides the most natural representation. We will thus discuss in the following the residual interaction in momentum space. In general, the effective interaction kernel is an involved fourpoint function, depending on three momenta: $\mathbf{p}$ and $\mathbf{p}^{\prime}$ as the momenta of the in-coming and out-going hole states and $\mathbf{q}$ as the transferred momentum. Figure 1 illustrates
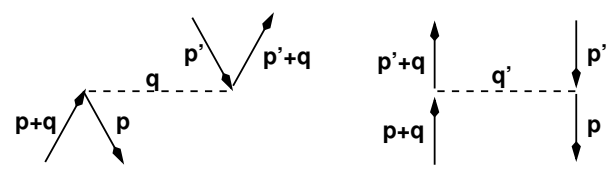

FIG. 1. Graphical representation of a local interaction in $p h$ space with direct (left) and exchange term (right). Particle and hole states are represented by full lines with up- and down arrows. The dashed horizontal line stands for the interaction. The three relevant momenta $\mathbf{p}, \mathbf{p}^{\prime}$, and $\mathbf{q}$, are indicated. The exchange term transfers the momentum $\mathbf{q}^{\prime}=\mathbf{p}^{\prime}-\mathbf{p}$.

these momenta for the case of a local interaction which is sufficient for our purposes because the Landau-Migdal as well as the Skyrme interaction are both local. All $p h$ pairs carry net momentum q. They differ by the other momentum $\mathbf{p}$, or $\mathbf{p}^{\prime}$ respectively. In Landau approximation, one considers the residual interaction $F^{p h}$ at the Fermi surface (we use here the symbol $F$ to distinguish Landau-Midgal approach from $V$ in SHF). Thus one can approximate it as a local contact (zero-range) interaction 
and $F^{p h}$ depends not on the $\mathbf{q}$ any more, only on the angle between the momenta $\mathbf{p}$ and $\mathbf{p}^{\prime}$ before and after the collision; its spin-independent part reads

$$
\begin{aligned}
F^{p h}\left(\frac{\mathbf{p} \cdot \mathbf{p}^{\prime}}{p_{F}^{2}}\right) & =C_{0} \sum_{l=0}^{\infty}\left[f_{l}+f_{l}^{\prime} \hat{\tau_{\mathbf{1}}} \cdot \hat{\tau_{\mathbf{2}}}\right] P_{l}\left(\frac{\mathbf{p} \cdot \mathbf{p}^{\prime}}{p_{F}^{2}}\right) \\
C_{0} & =\frac{\pi^{2} \hbar^{3}}{2 m^{*} p_{\mathrm{F}}} \quad, \quad p_{\mathrm{F}}=\hbar\left(\frac{3 \pi^{2} \rho_{0}}{2}\right)^{1 / 3}
\end{aligned}
$$

where $P_{l}(x)$ is the Legendre polynomial of order $l$ and $p_{F}$ is the Fermi momentum. There are, in fact, four terms containing different combinations of spin and isospin operators covering the typical nuclear interaction channels [36]. We consider here only the two terms which are relevant for modes with natural parity as, e.g., the giant resonance. By virtue of the Landau quasi-particle concept, the whole information content of the two-body interaction in matter shrinks to a few model constants, the much celebrated Landau-Migdal (dimensionless) parameters $f_{l}$. The scaling factor $C_{0}$ is proportional to the density of states at the Fermi surface. A typical value is $C_{0}=150 \mathrm{MeV} \mathrm{fm}^{3}$ which is the standard choice in phenomenological shell models where the effective mass is $m^{*} / m=1$. The importance of the $f_{l}$ shrinks with increasing $l$. Usually, only $l=0$ and 1 are taken into account. The Fourier transforms of the terms with $l=0$ and $l=1$ yield $\delta$-functions in coordinate space and derivatives thereof, a form which resembles very much the Skyrme force as given in Eq. A5). This already indicates that there is close relation between Landau-Migdal theory and SHF.

In the Theory of Fermi Liquids the Landau parameters are constants. Migdal introduced in his Theory of Finite Fermi Systems density dependent parameters $f_{l}(\rho)$ in order to correct for the finite size of the nuclei. The density dependent Landau-Migdal parameters are parametrized as [17]:

$$
f(\rho)=f^{(\mathrm{ex})}+\left(f^{(\mathrm{in})}-f^{(\mathrm{ex})}\right) \frac{\rho_{0}(r)}{\rho_{0}(0)}
$$

where $f^{(\mathrm{ex})}$ stands for the exterior region of the nucleus and $f^{\text {(in) }}$ for the interior. The Landau-Migdal interaction describes one part of the RPA scheme, the residual interaction. In Landau-Migdal theory, the ground-state input (s.p.-wavefunctions, s.p. energies) is taken from an empirical single-particle model which reproduces experimental s.p. energies as good as possible. The LandauMigdal parameters are free parameters of the model and tuned to generally accepted values of NMP, incompressibility, symmetry energy and effective masses, for details see [6].

SHF goes one step further in that it describes ground states as well as excitation properties with one and the same energy functional. The residual interaction for RPA calculations is derived with Eq. (3), for details see appendix A5-A9. The terms for natural-parity modes (no spin-spin interaction) eventually read in momentum space

$$
\begin{aligned}
F_{\mathrm{Sk}}^{p h}\left(\mathbf{p}, \mathbf{p}^{\prime}, \mathbf{q}\right)= & a_{00}+a_{01}\left(\boldsymbol{\tau} \boldsymbol{\tau}^{\prime}\right)+a_{z}\left(\tau_{z}+\tau_{z}^{\prime}\right) \\
& +\left[b_{00}^{(-)}+b_{01}^{(-)}\left(\boldsymbol{\tau} \boldsymbol{\tau}^{\prime}\right)\right] \mathbf{q}^{2} \\
& +\left[b_{00}^{(+)}+b_{01}^{(+)}\left(\boldsymbol{\tau} \boldsymbol{\tau}^{\prime}\right)\right]\left(\mathbf{p}-\mathbf{p}^{\prime}\right)^{2}, \\
a_{0 T}= & \frac{1}{4} \sum_{\tau}\left[F_{\tau, \tau}^{0}+(-1)^{T} F_{\tau,-\tau}^{0}\right], \\
a_{z}= & \frac{1}{4} \sum_{\tau}\left(\tau_{z}\right)_{\tau, \tau} F_{\tau, \tau}^{0}
\end{aligned}
$$

where $F_{\tau, \tau}^{0}, F_{\tau,-\tau}^{0}(\tau=n, p)$ and the $b$ parameters are given in appendix A5. This is the residual interaction as it must be taken into account in a consistent SkyrmeRPA calculation of modes with natural parity. It is interesting to check the expression in Landau approximation which reads in the limit of nuclear matter

$$
\boldsymbol{q}=0 \quad, \quad \mathbf{p}^{2}=\mathbf{p}^{\prime 2}=k_{F}^{2} .
$$

yielding

$$
\boldsymbol{q}^{\prime 2}=2 k_{F}^{2}\left[1-P_{1}(\cos \theta)\right] \quad, \quad \cos \theta=\frac{\mathbf{p} \cdot \mathbf{p}^{\prime}}{k_{F}^{2}} .
$$

This erase the direct term $\propto \mathbf{q}^{2}$ leaving for the momentum dependent part

$$
\begin{aligned}
F_{\text {Lan,grad }}^{p h}= & 2 k_{F}^{2}\left[b_{00}^{(+)}+b_{01}^{(+)}\left(\boldsymbol{\tau} \boldsymbol{\tau}^{\prime}\right)\right] \\
& -2 k_{F}^{2}\left[b_{00}^{(+)}+b_{01}^{(+)}\left(\boldsymbol{\tau} \boldsymbol{\tau}^{\prime}\right)\right] P_{1}(\cos \theta)
\end{aligned}
$$

Note that the velocity dependent exchange terms contribute to the leading order of $F^{p h}\left(f_{0}, f_{0}^{\prime}\right)$ as well as to the next to leading order $\left(f_{1}, f_{1}^{\prime}\right)$. Comparing Eq. (21) with the full form 18a, we see that, in spite of much similarity, the Landau-Migdal approximation modifies the residual interaction in detail. The effect of momentum dependence is formally obvious from Eq. (21). The effect of density-dependence is illustrated in figure 2 and shows the dimensionless Landau-Migdal parameters $f_{0}(\rho)$ and $f_{0}^{\prime}(\rho)$ for the SHF parametrization SV-bas and compares it with the standard linear Landau-Migdal form (17). The $f_{0}$ and $f_{0}^{\prime}$ parameters look, at first glance, much different from the linear trend the Landau-Migdal ansatz. However, one has to remind that nuclear resonance excitations do not explore the whole range of densities. The dynamically most relevant region lies between volume density and density at the surface $\rho \approx 0.1-0.16$ $\mathrm{fm}^{-3}$ and here, the differences are not so dramatic. The results from an RPA calculation in Landau-Migdal approximation are basically fine 37, 38. But for a detailed description, one should use the full SHF residual interaction. This has to include also a correct residual interaction from the other terms in the functional, particularly concerning the Coulomb interaction [39]. The density dependence of the $l=1$ parameters, $\mathrm{f}_{1}$ and $\mathrm{f}_{1}^{\prime}$, causes no problem as it is also linear in SHF which is obvious from figure 2 . 
$\mathrm{T}=1$ Landau parameters
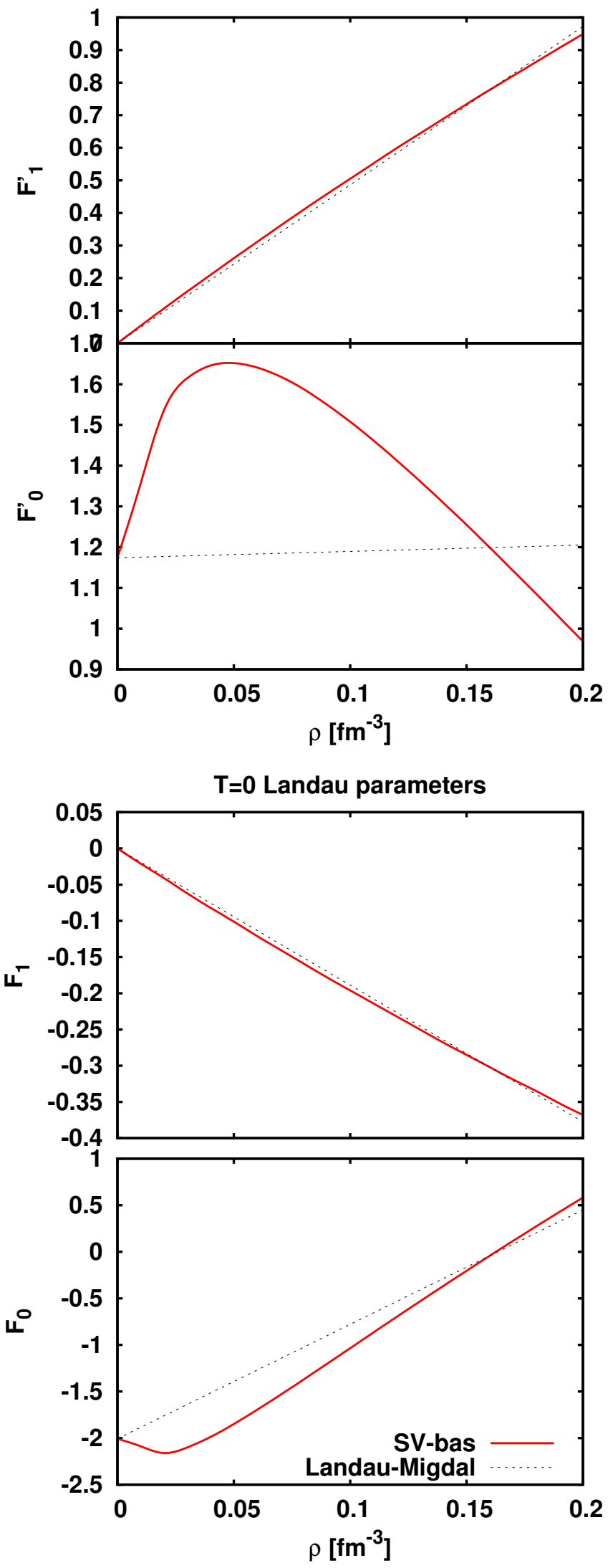

FIG. 2. Dimensionless Landau-Migdal parameters for the Skyrme parametrization SV-bas 28 together with the linear approximation according to the Landau-Migdal ansatz (17).

\section{RESULTS}

From the huge variety of possible results, we concentrate on the three most important giant resonances: the isoscalar giant monopole resonance (GMR), the isoscalar giant quadrupole resonance (GQR), and the isovector giant dipole resonance (GDR). To avoid interference with pairing effects, we confine the study to the doubly magic nuclei ${ }^{16} \mathrm{O},{ }^{40} \mathrm{Ca},{ }^{48} \mathrm{Ca}$, and ${ }^{208} \mathrm{~Pb}$.

\section{A. Details of the calculation scheme}

Eqs. (5) and (12) for the response functions in our approach are solved in a discrete basis. This basis is defined as a set of solutions of the Schrödinger equation with box boundary conditions. Both equations are solved in the same large configuration space. The RPA solutions with sufficiently strong $B(E L)$ values are taken as the phonons for the TBA calculation. We check stability of the results with phonon space and show here only the converged results. A detailed discussion of stability with respect to size of RPA space, phonon space, and $B(E L)$ cutoff will be postponed to a forthcoming publications. Actually, we use for phonon coupling all RPA modes which exhaust more than $20 \%$ of the total $B(E L)$ strength. For final TBA and RPA results we switch to a description of the nucleon continuum by using the continuum representation for the free $1 p 1 h$ response propagator $R^{(0)}$ in eqs. (5) and 12, details will be presented in a separate publication. It is to be noted that continuum effects are marginal for ${ }^{208} \mathrm{~Pb}$, but play a significant role for the lighter nuclei in the survey. The residual interaction $V$ in Eqs. (5) and (12) is derived fully self-consistently from the $\mathrm{SHF}$ functional according to Eq. (3). In the case of the energy density functional $E[\rho]$ built on the Skyrme forces, the amplitude $V$ determined by Eq. (3) contains the zero-range (velocity-independent), and velocitydependent parts, and the Coulomb interaction. Explicit formulas for all these terms of $\mathrm{V}$ are given in Appendices A5-A9.

We will consider only the doubly-magic nuclei. They have closed shells and pairing is not important. The box sizes in the RPA and TBA calculations are $15 \mathrm{fm}$ for ${ }^{16} \mathrm{O},{ }^{40,48} \mathrm{Ca}$ and $18 \mathrm{fm}$ for ${ }^{208} \mathrm{~Pb}$. The cutoff for the $1 p 1 h$ space is $100 \mathrm{MeV}$ for all nuclei (see our discussion in the next two sections). The new aspects in the present calculations as compared to earlier presentation are:

1) full residual interaction from the SHF functional,

2) continuum effects, and

3) subtraction method 13 in TBA.

Point 1 , the fully residual interaction, assures consistency of the calculations. Point 2, the particle continuum, serves to model correctly the escape with in the spectrum. Point 3, the subtraction of the static contribution from the $1 p 1 h$-phonon-interaction eliminates the double counting, resolves the stability problem of TBA, and reinstates the Thouless theorem, which otherwise does not 


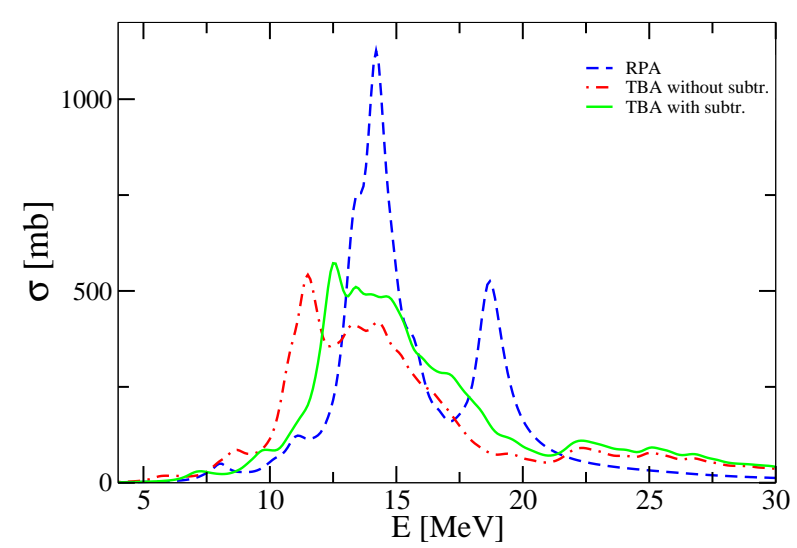

FIG. 3. Photo-absorption strength in ${ }^{208} \mathrm{~Pb}$ calculated with the parameter set SV-m64k6. Compared are RPA, plain TBA, and TBA with subtraction scheme (13). Details of the calculations are the same as described in section IVA with spectral smoothing parameter $\Delta=400 \mathrm{keV}$.

hold for extended versions of the RPA.

\section{B. The impact of the subtraction scheme}

First, we have a look at the effect of the subtraction scheme (13) for the residual interaction in TBA. Figure 3 demonstrates that for the photo-absorption strength in ${ }^{208} \mathrm{~Pb}$. The RPA spectrum looks already rather smooth due to the high density of $1 p 1 h$ states, continuum treatment, and folding with $\Delta=400 \mathrm{keV}$. But there is a pronounced secondary peak at higher energy around 18 $\mathrm{MeV}$ which is not found in experimental data (see figure 4). The coupling to complex configurations in TBA smooths the unnatural high-energy peak and turns it to a long high-energy tail in the spectrum which is also found in the experimental spectra. This means that TBA is correctly describing collisional broadening and both version of TBA do that in similar manner. Besides broadening, TBA induces also an energy shift in the spectra, usually a down shift as seen here. This shift is much reduced by the subtraction scheme and that is a desirable effect. An example for this is the isoscalar quadrupole channel in ${ }^{208} \mathrm{~Pb}$. There is a strong low lying $2_{1}^{+}$mode around 4 $\mathrm{MeV}$ and one expects that it is robust against complex configurations because the phase space or collisional effects is too small at this low energy. It turns out that this mode is heavily down shifted for plain TBA but almost inert, as it should be, for TBA with subtraction scheme. Altogether, we see that the subtraction of the zero-frequency interaction $W(0)$ is a crucial ingredient in TBA.

\section{Strength distributions}

In Fig. 4 the theoretical cross sections of GMR, GQR and GDR are compared with the experimental ones for ${ }^{208} \mathrm{~Pb}$. The theoretical results are calculated with all seven Skyrme parameter sets which we presented in Table I of section III A. We first discuss the GMR (left column) which is closely connected with the incompressibility $K$. The peak position is clearly related to $K$. Low $K$ (upper left panel) shifts the peak to lower energy while high $K$ shift it up. All parametrizations with $K=234 \mathrm{MeV}$ (left column, panels $2-5$ from above) produce the GMR at the same and correct place although they differ in other NMP. The GQR is shown in the middle column. It confirms what had been found earlier [42, namely that the GQR depends sensitively on the effective mass $m^{*} / m$ with the peak position going up with smaller $m^{*} / m$. RPA fits best with $m^{*} / m=0.9$ (panels 1-3 and 5 from above) but misses the high-energy tail. TBA provides best results with $m^{*} / m=0.7$ and produces properly the upper tail of the spectral distribution. The GDR is shown on the right column. Most prominent is the unphysical high-energy peak which shows up for all parametrizations and the welcome feature that TBA removes it consistently, as was discussed in section IVB. What trends is concerned, we see for the GDR the strongest impact coming from $\kappa_{\mathrm{TRK}}$, see the deviation for SV-kap00 (panel 3 from above). The situation is mixed for SV-m64k6 and SV-m56k6 because more than one NMP was varied (see table I).

Fig. 5 shows photo-absorption cross section (isovector dipole channel) and multipole strength distributions (isoscalar channels) for ${ }^{48} \mathrm{Ca}$. The general trends are much the same as for ${ }^{208} \mathrm{~Pb}$. New is that in lighter nuclei spectra become broader due to more spectral fragmentation. A closer inspection of the nucleus ${ }^{48} \mathrm{Ca}$ may help to assess the importance of the phonon degree of freedom in theoretical approaches to the spectral strength distributions. We first look at the GDR strength (right column). The experimental GDR strength is centered at $20.2 \mathrm{MeV}$ and shows large and smooth low- and highenergy tails, see Fig. 5. The mean-field approximation(RPA) produces a fragmentation of the strength into two major peaks and overestimates the height of the peaks by approximately a factor 2 . The detailed shape of the strength distribution is sensitive to the chosen parametrization of the effective interaction. Using a small effective mass, significant RPA strength is produced at a high energy of $25 \mathrm{MeV}(\mathrm{SV}-\mathrm{m} 56 \mathrm{~K} 6)$. The phonon coupling in TBA mainly reduces the strength in the vicinity of the two RPA peaks and re-distributes it. The effect of TBA is most pronounced for the interaction SV-m56K6 where the final TBA strength comes closest to the experimental data. The effect of peak reduction and broadening is more pronounced for the GQR strength (middle column) where RPA shows a too narrow resonance that dramatically overestimates the experimental strength of the peak. The phonon coupling reduces the strength of 
${ }^{208} \mathrm{~Pb} \quad \mathrm{~L}=0, \mathrm{~T}=0$

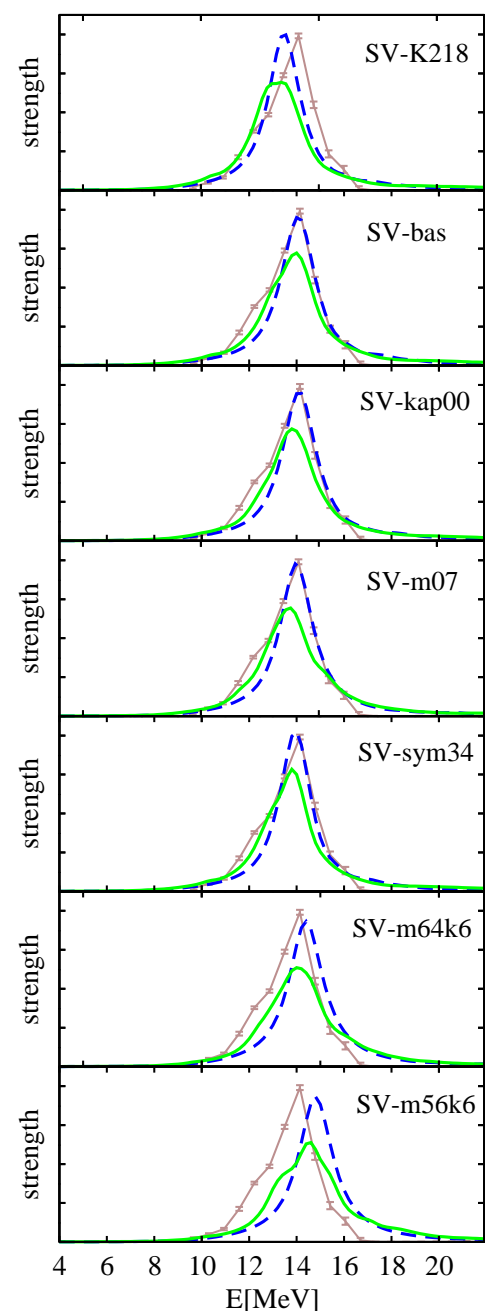

${ }^{208} \mathrm{~Pb} \quad \mathrm{~L}=2, \mathrm{~T}=0$

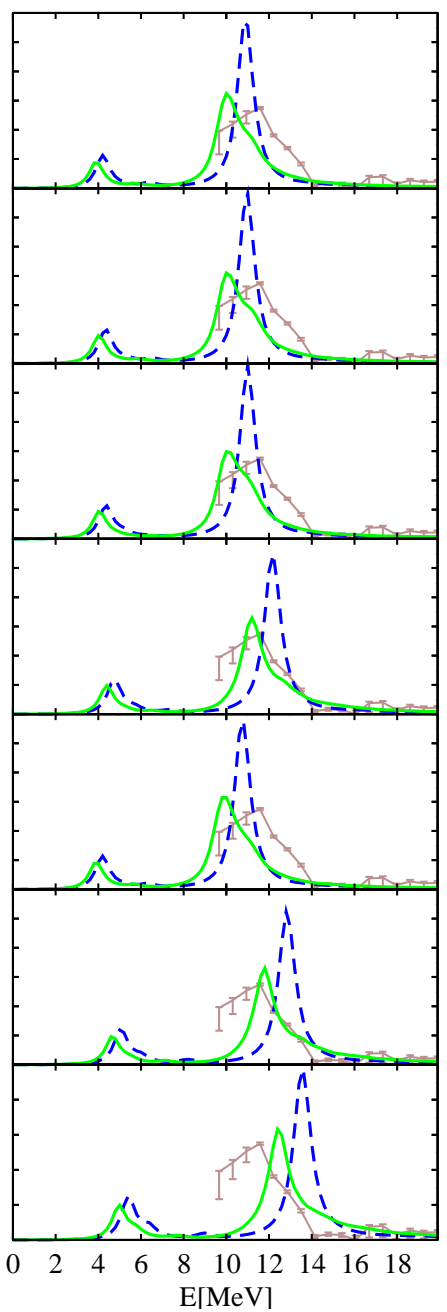

${ }^{208} \mathrm{~Pb} \quad \mathrm{~L}=1, \mathrm{~T}=1$

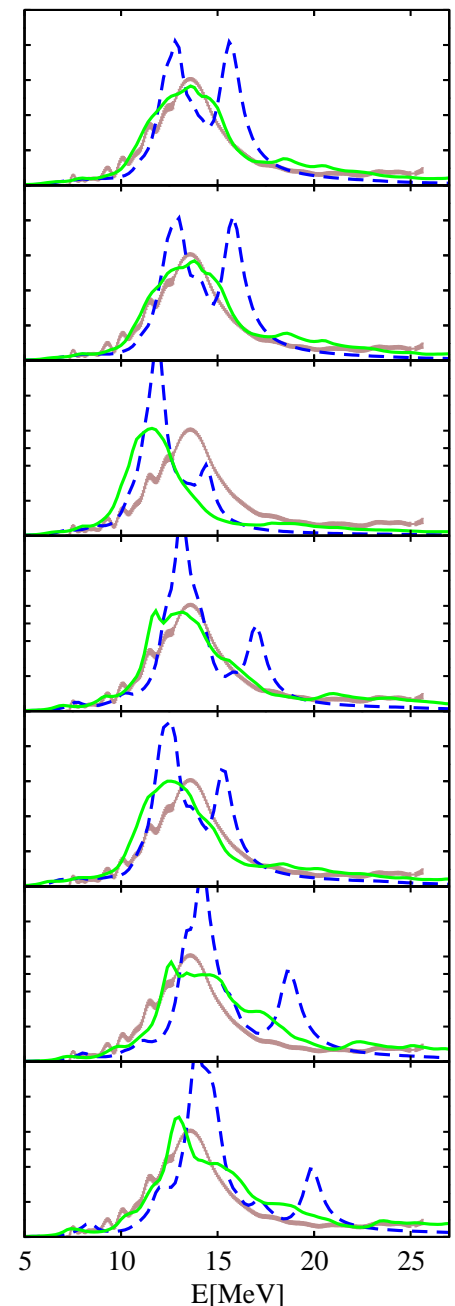

FIG. 4. Detailed spectral strength distributions for ${ }^{208} \mathrm{~Pb}$ and the three modes under consideration: isoscalar monopole (left panels), isoscalar quadrupole (middle panels), and isovector dipole (right panels). Photo-absorption strength is shown in case of the dipole mode, multipole strength else-wise. Compared are results from RPA (blue dashed) and TBA (green) with experimental strengths (brown with errorbars). Results are obtained with the seven Skyrme parametrizations presented in section III A. Experimental data are from 40, for the GDR and 41] for GMR and GQR.

the RPA peak by a factor 2. Even this does not yet suffice to obtain a quantitative description of the experimental quadrupole data. Somehow, collisional broadening is still underestimated. This underestimation is even more dramatic for the GMR (left column) where phonon coupling in TBA makes only a minor modification of the RPA result. From a formal point of view, this is plausible because the $\mathrm{L}=0$ channel limits the possible phonon couplings. Experimental data, however, are significantly broadened, more than for the other modes. It seems that TBA is not properly accounting for the crucial broadening mechanisms in ${ }^{48} \mathrm{Ca}$ (and other light nuclei). This leaves an open problem for future research.

The main result from the large collection of strengths shown in this section is that the modifications of the RPA results brought in by TBA (broadening and shift) are for a given channel and nucleus are practically the same for all parametrizations. We will see this also from the compact analysis in the next section.

\section{Trends in terms of peak energies}

After looking at strength functions in detail, we want to summarize here the net effect of TBA in terms of one key number. To that end, we define a resonance peak energy by averaging the strength in a window around the resonance. The peak energy was defined as the energy centroid $m_{1} / m_{0}$ where the moments $m_{1}$ and $m_{0}$ were taken in a certain energy interval around the resonance peak. These windows are $11<E^{*}<40 \mathrm{MeV}$ for GMR and GQR in ${ }^{16} \mathrm{O}, 15<E^{*}<30 \mathrm{MeV}$ for the 

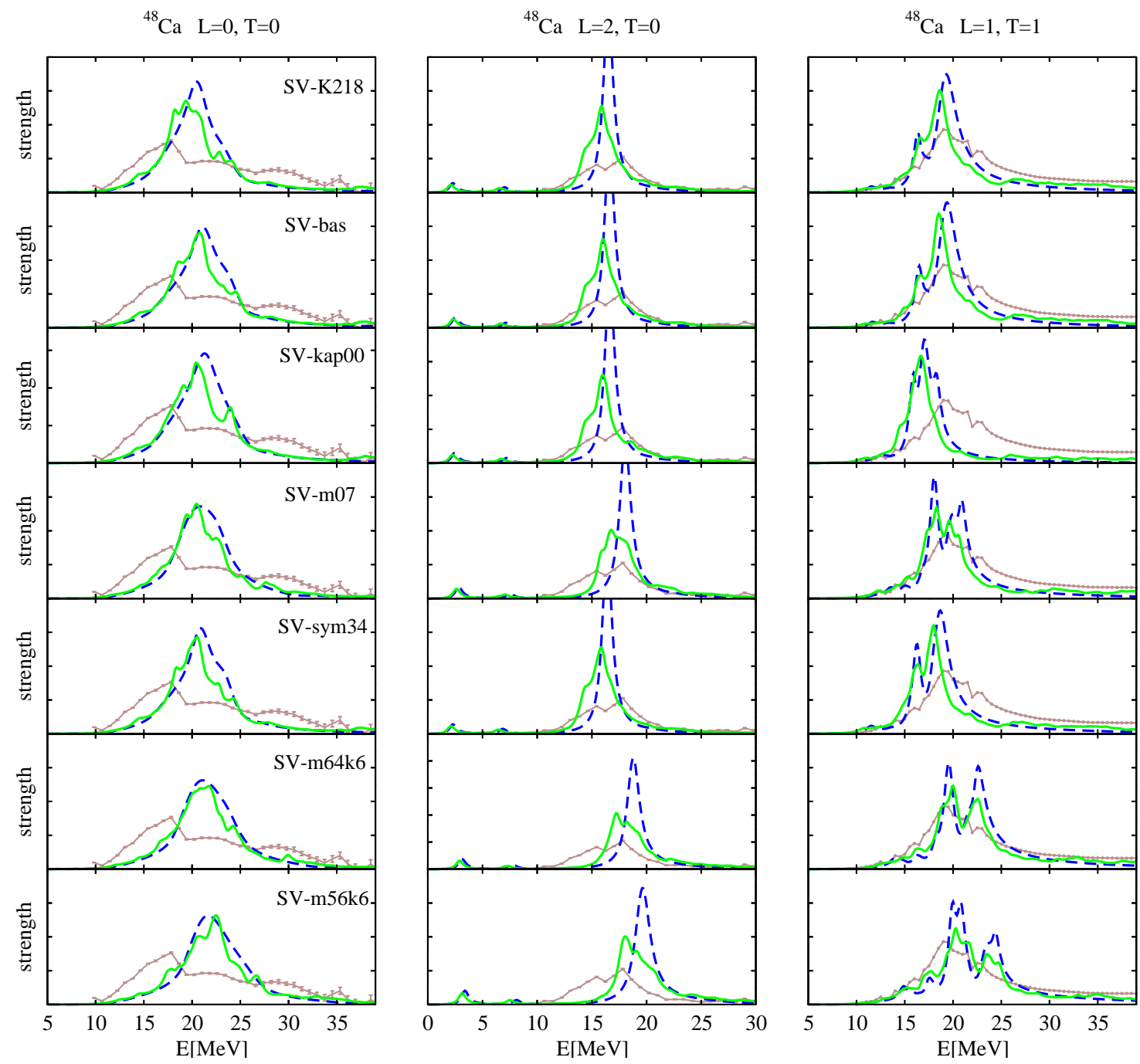

FIG. 5. As Fig. 4 but for ${ }^{48}$ Ca. Experimental data are taken from [4] for the GDR and 44 for GMR and GQR.

\begin{tabular}{|l|lll|llll|lll|}
\hline & \multicolumn{3}{|c|}{ GDR } & \multicolumn{3}{c|}{ GMR } & \multicolumn{3}{c|}{ GQR } \\
& exp. & RPA & TBA & exp. & RPA & TBA & exp. RPA & TBA \\
\hline${ }^{16} \mathrm{O}$ & 24.3 & 20.8 & 19.7 & 21.1 & 23.1 & 22.4 & 19.8 & 20.1 & 19.9 \\
${ }^{40} \mathrm{Ca}$ & 20.4 & 19.0 & 17.3 & 18.7 & 21.1 & 20.4 & 17.3 & 16.6 & 16.3 \\
${ }^{48} \mathrm{Ca}$ & 20.2 & 19.3 & 18.5 & 19.0 & 20.5 & 20.0 & 16.6 & 16.8 & 16.3 \\
${ }^{208} \mathrm{~Pb}$ & 13.5 & 14.3 & 13.6 & 13.8 & 14.0 & 13.8 & 11.5 & 10.9 & 10.4 \\
\hline
\end{tabular}

TABLE II. Peak energies for GDR, GMR, abd GQR in four doubly-magic nuclei computed with then parametrization SVbas. Compared are RPA an TBA results with the experimental value.

GDR in ${ }^{16} \mathrm{O}, 10<E<30 \mathrm{MeV}$ for GMR in ${ }^{40,48} \mathrm{Ca}$, and $10<E<25 \mathrm{MeV}$ for GQR in ${ }^{40,48} \mathrm{Ca}$, The centroids $E_{0}$ for the GDR in $40,48 \mathrm{Ca}$ and for the GDR, GMR, and GQR in $208 \mathrm{~Pb}$ were calculated in the window $E_{0} \pm 2 \delta$ where $\delta$ is the spectral dispersion (although with constraint $\delta \geq 2 \mathrm{MeV}$ ). Table II shows the results for the four nuclei and three resonance modes under considera- tion. We do that for one parametrization, SV-bas, only in order to concentrate on the trends with system size. The difference between RPA and TBA for the GDR is about the same for all four nuclei while the GMR shows a significant increase towards smaller nuclei and the GQR has the opposite trend to yield smaller difference for smaller systems. In any cases, the trends are not nearly as strong as they were in earlier calculations, see e.g. 77. The subtraction scheme $(13)$ tends to reduce the shift of resonance energies while maintaining full collisional broadening from phonon coupling. In the terms of many-body theory this means that the subtraction scheme reduces the effect on the real part of the resonance energy while maintaining the full effect on the imaginary part [45]. What the comparison with experimental results is concerned, we see acceptable agreement for ${ }^{208} \mathrm{~Pb}$. That is the nucleus where SV-bas was tuned to the resonances. Significant differences develop for lighter nuclei. This is a known problem for the GDR [46]. A thorough study of the $A$-dependence for the isoscalar modes has still to 


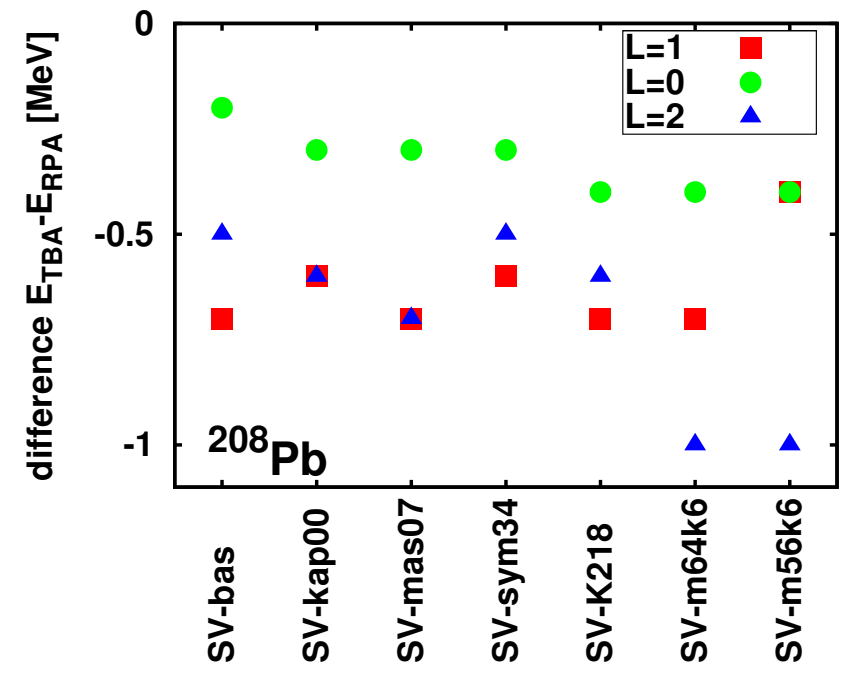

FIG. 6. Difference between RPA and TBA for the average energies of the three giant resonance modes in ${ }^{208} \mathrm{~Pb}$ for a variety of Skyrme parametrizations as indicated.

come.

Figure 6 shows the difference between RPA and TBA resonance energies for one nucleus ${ }^{208} \mathrm{~Pb}$, but now for all parametrizations under consideration. The GMR seems rather robust as it shows a small correction with little dependence on the parametrization. Sizable shifts are seen for GDR as well as GQR and these can vary significantly with parametrization. For the GQR, the trend is clear. The shift increases with decreasing effective mass $\mathrm{m}^{*} / \mathrm{m}$ and shows also some influence from the isovector effective mass characterized by $\kappa_{\text {TRK }}$. The GDR, again, seems to be rather robust, except for the one parametrization SVm56k6 for which many NMP had been changed.

\section{SUMMARY}

We have reviewed recent progess of the TBA, a selfconsistent generalization of the Landau-Migdal theory based on effective Skyrme interactions and incorporating the phonon degree of freedom. Phonons are shown to be a relevant degree of freedom in theoretical approaches to the nuclear multipole strength functions. In heavy nuclei, a major improvement with respect to a mean-field approximation(RPA) is obtained. The performance of the method deteriorates in light nuclei, however, where phonons by themselves do not suffice to account for the experimentally observed fragmentation of the strength. Other degrees of freedom appear to be important which limits the method to heavy nuclei. As the method is based on the effective Skyrme interaction which is valid for all nuclei, extrapolations to heavy neutron-rich nuclei are possible.

\section{ACKNOWLEDGMENTS}

This work has been supported by contract Re32213/1from the DFG.

\section{Appendix A: The Skyrme energy functional}

\section{Basic constituents: Densities and currents}

In SHF, a system is described in term of a set of single particle (s.p.) wavefunctions $\varphi_{\alpha}(\vec{r})$ together with BCS amplitudes $v_{\alpha}$ for occupation and $u_{\alpha}=\sqrt{1-v_{\alpha}^{2}}$ for nonoccupation. These are summarized in the one-body density matrix

$$
\varrho_{q}\left(\mathbf{r}, \mathbf{r}^{\prime}\right)=\sum_{\alpha \in q} w_{\alpha} v_{\alpha}^{2} \varphi_{\alpha}(\mathbf{r}) \varphi_{\alpha}^{\dagger}\left(\mathbf{r}^{\prime}\right)
$$

where $q$ labels the nucleon species with $q=p$ for protons and $q=n$ for neutrons. The $w_{\alpha}$ is a further factor which describes a cutoff for pairing space (see below). The SHF functional requires the knowledge of only a few local densities and and currents, sorted here according to time parity:

$$
\begin{array}{ll}
\text { time even: } & \\
\rho_{q}=\left.\operatorname{tr}_{\sigma}\left\{\varrho\left(\mathbf{r}, \mathbf{r}^{\prime}\right)\right\}\right|_{r=r^{\prime}} & \equiv \text { density } \\
\tau_{q}=\left.\operatorname{tr}_{\sigma}\left\{\nabla_{r} \nabla_{r^{\prime}} \varrho\left(\mathbf{r}, \mathbf{r}^{\prime}\right)\right\}\right|_{r=r^{\prime}} & \equiv \text { kinetic density } \\
\vec{J}_{q}=-\left.\operatorname{itr} \sigma\left\{\nabla_{r} \times \hat{\vec{\sigma}} \varrho\left(\mathbf{r}, \mathbf{r}^{\prime}\right)\right\}\right|_{r=r^{\prime}} & \equiv \text { spin-orbit density }
\end{array}
$$

time odd:

$$
\begin{aligned}
\vec{\sigma}_{q}=\left.\operatorname{tr}_{\sigma}\left\{\hat{\vec{\sigma}} \varrho\left(\mathbf{r}, \mathbf{r}^{\prime}\right)\right\}\right|_{r=r^{\prime}} & \equiv \text { spin density } \\
\vec{j}_{q}=\Im\left\{\left.\operatorname{tr}_{\sigma}\left\{\nabla_{r} \varrho\left(\mathbf{r}, \mathbf{r}^{\prime}\right)\right\}\right|_{r=r^{\prime}}\right\} & \equiv \text { current } \\
\vec{\tau}_{q}=-\left.\operatorname{tr}_{\sigma}\left\{\hat{\vec{\sigma}} \nabla_{r} \nabla_{r^{\prime}} \varrho\left(\mathbf{r}, \mathbf{r}^{\prime}\right)\right\}\right|_{r=r^{\prime}} & \equiv \text { kinetic spin-dens. }
\end{aligned}
$$

time mixed:

$$
\xi_{q}=\sum_{\alpha \in q} w_{\alpha} u_{\alpha} v_{\alpha}\left|\varphi_{\alpha}\right|^{2} \quad \equiv \text { pairing density }
$$

It is advantageous to handle the densities in terms of isospin $T \in\{0,1\}$ instead of protons $p$ and neutrons $n$. Thus we consider the recoupled forms which read for the local density

$$
\rho_{0} \equiv \rho=\rho_{p}+\rho_{n} \quad, \quad \rho_{1}=\rho_{p}-\rho_{n} \quad,
$$

and similarly for the other densities and currents. The isoscalar density $\rho_{0} \equiv \rho$ is equivalent to the total density and the difference $\rho_{1}$ corresponds to the isovector density. All densities and currents in the collection (A2 are real and have definite time parity, except for the pairing density $\xi$ which is complex and has mixed time parity.

\section{The energy functional}

The total energy in SHF is composed from kinetic energy, Skyrme interaction energy, Coulomb energy, pairing 
energy, and correlation energy from low-energy collective modes, usually a center-of-mass and a rotational correlation. It reads

$$
\begin{aligned}
E_{\text {total }}= & \int d^{3} r\left\{\mathcal{E}_{\mathrm{kin}}+\mathcal{E}_{\mathrm{Sk}}\right\} \\
& +E_{\text {Coul }}+E_{\text {pair }}-E_{\mathrm{corr}} \\
\mathcal{E}_{\mathrm{kin}}= & \frac{\hbar^{2}}{2 m_{p}} \tau_{p}+\frac{\hbar^{2}}{2 m_{n}} \tau_{n} . \\
E_{\text {Coul }}= & \frac{e^{2}}{2} \int d^{3} r d^{3} r^{\prime} \frac{\rho_{p}(\vec{r}) \rho_{p}\left(\vec{r}^{\prime}\right)}{\left|\vec{r}-\vec{r}^{\prime}\right|} \\
& +\frac{3 e^{2}}{4}\left(\frac{3}{\pi}\right)^{1 / 3} \int d^{3} r\left[\rho_{p}(\vec{r})\right]^{4 / 3} .
\end{aligned}
$$

The pairing energy $E_{\text {pair }}$ is irrelevant for the present applications to doubly-magic nuclei. The correlation energy $E_{\text {corr }}$ amounts to a center-of-mass correction to groundstate observables and is ignored for the RPA excitations. The key piece is the Skyrme energy-density functional $\mathcal{E}_{\mathrm{Sk}}$ which can be sorted into time-even and time-odd couplings as

$$
\begin{array}{rlrl}
\mathcal{E}_{\text {Sk }, \text { even }}= & C_{0}^{\rho} \rho_{0}^{2} & +C_{1}^{\rho} \rho_{1}^{2} \\
& +C_{0}^{\rho, \alpha} \rho_{0}^{2+\alpha} & +C_{1}^{\rho, \alpha} \rho_{1}^{2} \rho_{0}^{\alpha} \\
& +C_{0}^{\Delta \rho} \rho_{0} \Delta \rho_{0} & +C_{1}^{\Delta \rho} \rho_{1} \Delta \rho_{1} \\
& +C_{0}^{\nabla J} \rho_{0} \nabla \cdot \vec{J}_{0} & +C_{1}^{\nabla J} \rho_{1} \nabla \cdot \vec{J}_{1} \\
& +C_{0}^{\tau} \rho_{0} \tau_{0} & & +C_{1}^{\tau} \rho_{1} \tau_{1} \\
& +C_{0}^{J} \vec{J}_{0}^{2} & & +C_{1}^{J} \vec{J}_{1}^{2} \\
\mathcal{E}_{\mathrm{Sk}, \text { odd }}= & & \\
& C_{0}^{\sigma} \vec{\sigma}_{0}^{2} & & +C_{1}^{\sigma} \vec{\sigma}_{1}^{2} \\
& +C_{0}^{\sigma, \alpha} \vec{\sigma}_{0}^{2} \rho_{0}^{\alpha} & +C_{1}^{\sigma, \alpha} \vec{\sigma}_{1}^{2} \rho_{0}^{\alpha} \\
+ & C_{0}^{\Delta \sigma} \vec{\sigma}_{0} \Delta \vec{\sigma}_{0} & +C_{1}^{\Delta \sigma} \vec{\sigma}_{1} \Delta \vec{\sigma}_{1} \\
+ & C_{0}^{\nabla J} \vec{\sigma}_{0} \cdot \nabla \times \vec{j}_{0} & +C_{1}^{\nabla J} \vec{\sigma}_{1} \cdot \nabla \times \vec{j}_{1} \\
& -C_{0}^{\tau} \vec{j}_{0}^{2} & & -C_{1}^{\tau} \vec{j}_{1}^{2} \\
& -\frac{1}{2} C_{0}^{J} \vec{\sigma}_{0} \cdot \vec{\tau}_{0} & -\frac{1}{2} C_{1}^{J} \vec{\sigma}_{1} \cdot \vec{\tau}_{1}
\end{array}
$$

Note that at some places the same coupling constants appear in time-even and time-odd part. That serves to guarantee Galilean invariance of the functional, proven for the case of equal nucleon masses in [47. Spin couplings $\propto C_{T}^{\sigma}, C_{T}^{\sigma \alpha}, C_{T}^{\Delta \sigma}$ play no role for the natural-parity modes considered here and are only listed for completeness.

\section{The Skyrme "force"}

Originally SHF was proposed as an effective interaction [19], later on coined as "Skyrme force". In this approach, the SHF energy functional is given as the expectation value of the Skyrme interaction $\hat{V}_{\text {Skyrme }}$ for a Slater state $|\Phi\rangle$ (more generally a BCS state), i.e.

$$
\int d^{3} r \mathcal{E}_{\mathrm{Sk}}=\left\langle\Phi\left|\hat{V}_{\text {Skyrme }}\right| \Phi\right\rangle
$$

with

$$
\begin{aligned}
\hat{V}_{\text {Skyrme }}= & \hat{V}^{m}+\hat{V}^{L S}+\hat{V}^{t} \\
\hat{V}^{m}= & t_{0}\left(1+x_{0} \hat{P}_{\sigma}\right) \delta\left(\mathbf{r}_{12}\right) \\
& +\frac{t_{3}}{6}\left(1+x_{3} \hat{P}_{\sigma}\right) \rho^{\alpha}\left(\mathbf{r}_{1}\right) \delta\left(\mathbf{r}_{12}\right) \\
& +\frac{t_{1}}{2}\left(1+x_{1} \hat{P}_{\sigma}\right)\left(\delta\left(\mathbf{r}_{12}\right) \hat{\boldsymbol{k}}^{2}+\hat{\boldsymbol{k}}^{\prime 2} \delta\left(\mathbf{r}_{12}\right)\right) \\
& +t_{2}\left(1+x_{2} \hat{P}_{\sigma}\right) \hat{\boldsymbol{k}}^{\prime} \delta\left(\mathbf{r}_{12}\right) \hat{\boldsymbol{k}} \\
\hat{V}^{L S}= & \mathrm{i}\left(W_{0}\left(1+x_{W} P_{\tau}\right)\left(\hat{\boldsymbol{\sigma}}_{1}+\hat{\boldsymbol{\sigma}}_{2}\right) \cdot \hat{\boldsymbol{k}}^{\prime} \times \delta\left(\mathbf{r}_{12}\right) \hat{\boldsymbol{k}}\right. \\
\mathbf{r}_{12}= & \mathbf{r}_{1}-\mathbf{r}_{2}, \quad \hat{P}_{\sigma}=\frac{1}{2}\left(1+\hat{\boldsymbol{\sigma}}_{1} \hat{\boldsymbol{\sigma}}_{2}\right), \\
\hat{\boldsymbol{k}}_{=} & -\frac{i}{2}\left(\vec{\nabla}_{1}-\vec{\nabla}_{2}\right) \\
& i \\
\hat{\boldsymbol{k}}^{\prime}= & \frac{i}{2}\left(\overleftarrow{\nabla}_{1}-\overleftarrow{\nabla}_{2}\right) .
\end{aligned}
$$

where $\hat{\boldsymbol{k}}$ acts to the right and $\hat{\boldsymbol{k}}^{\prime}$ to the left. Note that the rather involved, but usually ignored, tensor force has not been listed here. This "Skyrme force" was motivated in that form from a low momentum expansion of the density-matrix [20, 48] which yields the above terms where each parameter $t_{i}$ carries, in principle, some density dependence. For simplicity, one has decided to ignore density dependence, except for the zeroth order term which is augmented by a density dependence $\rho^{\alpha}$. We put the notion "force" in quotation mark because this object depends on the density which is produced by the wave function on which this force acts. This is not a standard two-body operator, but an effective force designed for building an expectation value with a mean-field state 46.

Each one of the two viewpoints, the energy functional A4 and the Skyrme "force" A5, has a natural set of parameters associated to the terms of the functional (the $C_{T}^{(\text {typ) }}$ ), or force (the $t_{i}$ ) respectively. There is a one-to- 
one correspondence between the two conventions:

$$
\begin{aligned}
C_{0}^{\rho} & =\frac{3}{8} t_{0}, \\
C_{1}^{\rho} & =-\frac{1}{4} t_{0}\left(\frac{1}{2}+x_{0}\right), \\
C_{0}^{\tau} & =\frac{3}{16} t_{1}+\frac{5}{16} t_{2}+\frac{1}{4} t_{2} x_{2}, \\
C_{1}^{\tau} & =-\frac{1}{8}\left[t_{1}\left(\frac{1}{2}+x_{1}\right)-t_{2}\left(\frac{1}{2}+x_{2}\right)\right], \\
C_{0}^{\Delta \rho} & =-\frac{9}{64} t_{1}+\frac{5}{64} t_{2}+\frac{1}{16} t_{2} x_{2}, \\
C_{1}^{\Delta \rho} & =\frac{1}{32}\left[3 t_{1}\left(\frac{1}{2}+x_{1}\right)+t_{2}\left(\frac{1}{2}+x_{2}\right)\right], \\
C_{0}^{\rho \alpha} & =\frac{1}{16} t_{3} \\
C_{1}^{\rho \alpha} & =-\frac{1}{24} t_{3}\left(\frac{1}{2}+x_{3}\right), \\
C_{0}^{\nabla J} & =-\frac{3}{4} t_{4} \\
C_{1}^{\nabla J} & =-\frac{1}{4} t_{4}, \\
C_{0}^{s} & =-\frac{1}{4} t_{0}\left(\frac{1}{2}-x_{0}\right), \\
C_{1}^{s} & =-\frac{1}{8} t_{0}, \\
C_{0}^{s T} & =-\frac{1}{8}\left[t_{1}\left(\frac{1}{2}-x_{1}\right)-t_{2}\left(\frac{1}{2}+x_{2}\right)\right], \\
C_{1}^{s T} & =-\frac{1}{16}\left(t_{1}-t_{2}\right), \\
C_{0}^{\Delta s} & =\frac{1}{32}\left[3 t_{1}\left(\frac{1}{2}-x_{1}\right)+t_{2}\left(\frac{1}{2}+x_{2}\right)\right], \\
C_{1}^{\Delta s} & =\frac{1}{64}\left(3 t_{1}+t_{2}\right), \\
C_{0}^{s \alpha} & =-\frac{1}{24} t_{3}\left(\frac{1}{2}-x_{3}\right), \\
C_{1}^{s \alpha} & =-\frac{1}{48} t_{3},
\end{aligned}
$$

There is one exception concerning the spin-orbit term. The energy functional (A4) allows for independent choice of $C_{0}^{s T}$ and $C_{1}^{s T}$. That is freedom which was recommended in 49 and regularly used later on. But the force (A5) ties these two terms together as seen in eq. A66.

The derivation of the reduced elements of RPA residual two-body interaction in appendix A 5 refers to the force aspect of SHF and thus formulates the matrix elements preferably in terms of the force parameters $t_{i}$.

\section{Nuclear matter parameters}

Infinite nuclear matter is taken without Coulomb force, pairing, and correlation correction. It remains the energy per particle as

$$
\frac{E}{A}\left(\rho_{0}, \rho_{1}, \tau_{0}, \tau_{1}\right)=\frac{\mathcal{E}_{\text {kin }}+\mathcal{E}_{\mathrm{Sk}}}{\rho_{0}} .
$$

where we consider for a while $\rho$ and $\tau$ as independent variables. Of course, a given system is characterized just by the densities $\rho_{T}$ while the kinetic density depends on these given densities as $\tau_{T}=\tau_{T}\left(\rho_{0}, \rho_{1}\right)$. Thus we have to distinguish between partial derivatives $\partial / \partial_{\tau}$ which take $\tau_{T}$ as independent and total derivatives $d / d \rho$ which deal only with $\rho_{T}$ dependence. The relation is

$$
\frac{d}{d \rho_{T}}=\frac{\partial}{\partial \rho_{T}}+\sum_{T^{\prime}} \frac{\partial \tau_{T^{\prime}}}{\partial \rho_{T}} \frac{\partial}{\partial \tau_{T^{\prime}}} .
$$

The standard NMP are defined at the equilibrium point $\left(\rho_{0}=\rho_{\text {eq }}, \rho_{1}=0\right)$ of symmetric nuclear matter. They isoscalar ground state properties

equilibrium density: $\left.\quad \rho_{\mathrm{eq}} \leftrightarrow \frac{d}{d \rho_{0}} \frac{E}{A}\right|_{\text {eq }}=0$

equilibrium energy: $\left.\frac{E}{A}\right|_{\text {eq }}$

isoscalar response properties

$\begin{array}{ll}\text { incompressibility: } & K_{\infty}=\left.9 \rho_{0}^{2} \frac{d^{2}}{d \rho_{0}^{2}} \frac{E}{A}\right|_{\mathrm{eq}} \\ \text { effective mass: } & \frac{\hbar^{2}}{2 m *}=\frac{\hbar^{2}}{2 m}+\left.\frac{\partial}{\partial \tau_{0}} \frac{E}{A}\right|_{\mathrm{eq}}\end{array}$

isovector response properties

\begin{tabular}{ll}
\hline symmetry energy: $\quad J$ & $=\left.\frac{1}{2} \rho_{0}^{2} \frac{d^{2}}{d \rho_{1}^{2}} \frac{E}{A}\right|_{\mathrm{eq}}$ \\
slope of $J:$ & $L=3 \rho_{0} \frac{d}{d \rho_{0}} J$ \\
TRK sum-rule enh.: $\kappa_{\mathrm{TRK}}$ & $=\left.\frac{2 m}{\hbar^{2}} \frac{\partial}{\partial \tau_{1}} \frac{E}{A}\right|_{\mathrm{eq}}$ \\
\hline
\end{tabular}

TABLE III. Definition of the nuclear matter properties (NMP). All derivatives are to be taken at the equilibrium point of symmetric nuclear matter. For the definition of $L, J$ is considered temporarily as $J\left(\rho_{0}\right)$.

are summarized in table III. The enhancement factor for the Thomas-Reiche-Kuhn (TRK) sum rule 36] is a widely used way to characterize the isovector effective mass which is obvious from the given expression involving derivative with respect to $\tau_{1}$. The slope of symmetry energy $L$ characterizes the density dependence of the symmetry energy which allows to estimate the symmetry energy at half density, i.e. at surface of finite nuclei.

The NMP can be grouped into four classes: first, the (isoscalar) ground state properties $\rho_{\mathrm{eq}}$ and $E /\left.A\right|_{\mathrm{eq}}$, second, isoscalar response properties $K$ and $m / m$, and third, isovector response properties $J, L, \kappa_{\mathrm{TRK}}$. The response properties determine zero sound in matter [50] and subsequently they are closely related to giant resonance modes in finite nuclei as we will see later. There is a further category, the surface energies which go already beyond homogeneous matter and whose definition is rather involved [51]. They are not considered here.

Homogeneous matter yields $\Delta \rho=0$ and $\vec{J}=0$ which, in turn, renders four terms in the functiona A4e inactive. Thus we have exactly seven interaction parameters $\left(C_{0}^{\rho}, C_{0}^{\rho, \alpha}, C_{0}^{\tau}, \alpha\right)$ to determine seven NMP. The relation is revertible establishing a one-to-one correspondence between the both sets. This allows to consider the NMP equivalently as model parameters which is, in fact, a more intuitive way to communicate the model parameters. And this is the aspect which is used in the 
systematic variation of the SHF functional (see section III A.

\section{Reduced matrix elements of the residual interaction}

In this Appendix we draw the exact formulas for the reduced matrix elements of the residual interaction $V$ deduced from the Skyrme energy functional of the standard form (see, e.g., [10, 52]).

It is convenient to present this interaction as a sum of the following terms

$$
V_{12,34}=V_{12,34}^{(0)}+V_{12,34}^{(2) D}+V_{12,34}^{(2) X}+V_{12,34}^{(2) S O}+V_{12,34}^{(C)},
$$

where $V_{12,34}^{(0)}$ is the momentum-independent part of $V$ (including density-dependent terms), $V_{12,34}^{(2) D}, V_{12,34}^{(2) X}$ and $V_{12,34}^{(2) S O}$ are the direct, exchange and spin-orbit terms of the momentum-dependent part of the interaction (all these terms are of the second order in the momenta, see Ref. 35] for the explicit definition of $V_{12,34}^{(2) D}$ and $\left.V_{12,34}^{(2) X}\right)$, $V_{12,34}^{(C)}$ is the Coulomb interaction. It is supposed that the matrix elements in Eq. A9 are calculated in the representation of the single-particle wave functions $\varphi_{1}(\boldsymbol{r}, \sigma, \tau)$ of some spherically-symmetric basis. In this case the matrix indices can be represented as $1=\left\{(1), m_{1}\right\}$, where $(1)=\left\{\tau_{1}, n_{1}, l_{1}, j_{1}\right\}, m_{1}$ is a projection of the total angular momentum. Let us define reduced matrix elements as follows

$$
\begin{aligned}
V_{(12,34)}^{J} & =\sum_{m_{1} m_{2} m_{3} m_{4} M} V_{12,34} \\
& \times(-1)^{j_{2}-m_{2}}\left(\begin{array}{ccc}
j_{1} & j_{2} & J \\
m_{1} & -m_{2} & M
\end{array}\right) \\
& \times(-1)^{j_{4}-m_{4}}\left(\begin{array}{ccc}
j_{3} & j_{4} & J \\
m_{3} & -m_{4} & M
\end{array}\right) .
\end{aligned}
$$

For the excitations in the neutral particle-hole channel with the total angular momentum $J$ one obtains

$$
\begin{aligned}
V_{(12,34)}^{J} & =V_{(12,34)}^{J(0)}+V_{(12,34)}^{J(2) D}+V_{(12,34)}^{J(2) X} \\
& +V_{(12,34)}^{J(2) S O}+V_{(12,34)}^{J(C)} .
\end{aligned}
$$

Below the explicit formulas for the terms in this equality are given.

\section{Momentum-independent part of the interaction}

$$
\begin{aligned}
& V_{(12,34)}^{J(0)}=\frac{\delta_{\tau_{1}, \tau_{2}} \delta_{\tau_{4}, \tau_{3}}}{2 J+1} \sum_{L S} I_{(12,34)}^{S(0)} \\
& \times\left\langle j_{2} l_{2}\left\|T_{J L S}\right\| j_{1} l_{1}\right\rangle\left\langle j_{4} l_{4}\left\|T_{J L S}\right\| j_{3} l_{3}\right\rangle,
\end{aligned}
$$

where $S=0,1$

$I_{(12,34)}^{S(0)}=\int_{0}^{\infty} d r r^{2}\left[\delta_{S, 0} F_{\tau_{1}, \tau_{3}}^{0}(r)+\delta_{S, 1} G_{\tau_{1}, \tau_{3}}^{0}(r)\right]$

$\times R_{(1)}(r) R_{(2)}(r) R_{(3)}(r) R_{(4)}(r)$,

$R_{(1)}(r)$ is the radial part of the single-particle wave function $\varphi_{1}(\boldsymbol{r}, \sigma, \tau)$. The functions $F_{\tau, \tau^{\prime}}^{0}(r)$ and $G_{\tau, \tau^{\prime}}^{0}(r)$ are defined by the following equations

$$
\begin{gathered}
F_{n, n}^{0}=\frac{1}{2}\left(1-x_{0}\right) t_{0}+\frac{t_{3}}{48} \rho^{\alpha}\{3(\alpha+1)(\alpha+2) \\
\left.-\left(1+2 x_{3}\right)\left[\alpha(\alpha-1)(\bar{\rho} / \rho)^{2}+4 \alpha \bar{\rho} / \rho+2\right]\right\}(\mathrm{A} 14) \\
F_{n, p}^{0}=F_{p, n}^{0}=\left(1+x_{0} / 2\right) t_{0}+\frac{t_{3}}{48} \rho^{\alpha}\{3(\alpha+1)(\alpha+2) \\
\left.-\left(1+2 x_{3}\right)\left[\alpha(\alpha-1)(\bar{\rho} / \rho)^{2}-2\right]\right\}, \\
G_{n, n}^{0}=-\frac{1}{2}\left(1-x_{0}\right) t_{0}-\frac{t_{3}}{12}\left(1-x_{3}\right) \rho^{\alpha}, \quad(\mathrm{A} 16) \\
G_{n, p}^{0}=G_{p, n}^{0}=\frac{x_{0} t_{0}}{2}+\frac{x_{3} t_{3}}{12} \rho^{\alpha},
\end{gathered}
$$

where $t_{0}, x_{0}, t_{3}, x_{3}$, and $\alpha$ are the parameters of the Skyrme energy functional (see, e.g., 52]), $\rho=\rho_{n}+\rho_{p}$, $\bar{\rho}=\rho_{n}-\rho_{p}, \rho_{n}=\rho_{n}(r)$ and $\rho_{p}=\rho_{p}(r)$ are the neutron and proton local densities. Formulas for $F_{p, p}^{0}$ and $G_{p, p}^{0}$ are obtained from Eqs. A14 and A16 by replacing $\bar{\rho} \rightarrow$ $-\bar{\rho}$. $\left\langle j_{1} l_{1}\left\|T_{J L S}\right\| j_{2} l_{2}\right\rangle$ is the reduced matrix element of the spherical tensor operator $T_{J L S M}=\left(Y_{L} \otimes \sigma_{S}\right)_{J M}$ which is defined by the formula (see also Eqs. (A1)-(A4) of Ref. 53])

$$
\begin{aligned}
& \left\langle j_{1} l_{1}\left\|T_{J L S}\right\| j_{2} l_{2}\right\rangle \\
& =(-1)^{l_{1}} \sqrt{\frac{(2 J+1)(2 L+1)(2 S+1)}{2 \pi}} \\
& \times \sqrt{\left(2 j_{1}+1\right)\left(2 l_{1}+1\right)\left(2 j_{2}+1\right)\left(2 l_{2}+1\right)} \\
& \times\left(\begin{array}{ccc}
l_{1} & l_{2} & L \\
0 & 0 & 0
\end{array}\right)\left\{\begin{array}{ccc}
\frac{1}{2} & l_{2} & j_{2} \\
\frac{1}{2} & l_{1} & j_{1} \\
S & L & J
\end{array}\right\} .
\end{aligned}
$$

7. Direct and exchange terms of the momentum-dependent part of the interaction

$$
\begin{aligned}
& V_{(12,34)}^{J(2) D}=\frac{\delta_{\tau_{1}, \tau_{2}} \delta_{\tau_{4}, \tau_{3}}}{2 J+1} \sum_{S} C_{\tau_{1}, \tau_{3}}^{(S) D} U_{(12,34)}^{J S}, \\
& V_{(12,34)}^{J(2) X}=\delta_{\tau_{1}, \tau_{2}} \delta_{\tau_{4}, \tau_{3}} \sum_{S J^{\prime}} C_{\tau_{1}, \tau_{3}}^{(S) X} U_{(42,31)}^{J^{\prime} S} \\
& \times(-1)^{J+J^{\prime}+j_{1}-j_{4}}\left\{\begin{array}{ccc}
j_{2} & j_{4} & J^{\prime} \\
j_{3} & j_{1} & J
\end{array}\right\},
\end{aligned}
$$


where $S=0,1$,

$$
\begin{gathered}
U_{(12,34)}^{J S}=-\sum_{L} I_{(12,34)}^{L(2)} \\
\times\left\langle j_{2} l_{2}\left\|T_{J L S}\right\| j_{1} l_{1}\right\rangle\left\langle j_{4} l_{4}\left\|T_{J L S}\right\| j_{3} l_{3}\right\rangle, \quad(\mathrm{A} 21) \\
I_{(12,34)}^{L(2)}=\int_{0}^{\infty} d r\left[r^{2}\left(R_{(1)}(r) R_{(2)}(r)\right)^{\prime}\left(R_{(3)}(r) R_{(4)}(r)\right)^{\prime}\right. \\
\left.+L(L+1) R_{(1)}(r) R_{(2)}(r) R_{(3)}(r) R_{(4)}(r)\right], \quad(\mathrm{A} 22) \\
C_{\tau, \tau^{\prime}}^{(S) D}=-\left[b_{S 0}^{(-)}+\left(2 \delta_{\tau, \tau^{\prime}}-1\right) b_{S 1}^{(-)}\right], \\
C_{\tau, \tau^{\prime}}^{(S) X}=\frac{1}{2}\left\{b_{00}^{(+)}+\left(2 \delta_{\tau, \tau^{\prime}}-1\right) b_{01}^{(+)}\right. \\
\left.+(3-4 S)\left[b_{10}^{(+)}+\left(2 \delta_{\tau, \tau^{\prime}}-1\right) b_{11}^{(+)}\right]\right\} . \quad(\mathrm{A} 24)
\end{gathered}
$$

Normally, the parameters $b_{S T}^{( \pm)}$are expressed through the ordinary Skyrme-force parameters $t_{1}, x_{1}, t_{2}, x_{2}$ by the equations

$$
\begin{aligned}
& b_{00}^{( \pm)}=\frac{1}{16}\left[ \pm\left(5+4 x_{2}\right) t_{2}+3 t_{1}\right], \\
& b_{10}^{( \pm)}=\frac{1}{16}\left[ \pm\left(1+2 x_{2}\right) t_{2}-\left(1-2 x_{1}\right) t_{1}\right], \\
& b_{01}^{( \pm)}=\frac{1}{16}\left[ \pm\left(1+2 x_{2}\right) t_{2}-\left(1+2 x_{1}\right) t_{1}\right], \\
& b_{11}^{( \pm)}=\frac{1}{16}\left( \pm t_{2}-t_{1}\right) .
\end{aligned}
$$

If Eqs. A25- $\mathrm{A28}$ are fulfilled, we have

$$
V_{12,34}^{(2) X}=-V_{42,31}^{(2) D}=-V_{13,24}^{(2) D} .
$$

However, in the general case there is another way of the choice of these parameters in which they are expressed through the coupling constants of the Skyrme energy functional $C_{T}^{\tau}, C_{T}^{J}, C_{T}^{\Delta \rho}$, and $C_{T}^{\Delta s}$ (choice (ii) of Ref. [10). In this case we have

$$
\begin{aligned}
b_{0 T}^{(+)} & =C_{T}^{\tau}, \\
b_{1 T}^{(+)} & =-C_{T}^{J}, \\
b_{0 T}^{(-)} & =-2 C_{T}^{\Delta \rho}-\frac{1}{2} C_{T}^{\tau}, \\
b_{1 T}^{(-)} & =-2 C_{T}^{\Delta s}+\frac{1}{2} C_{T}^{J} .
\end{aligned}
$$

The inverse formulas read

$$
\begin{aligned}
C_{T}^{\Delta \rho} & =-\frac{1}{2} b_{0 T}^{(-)}-\frac{1}{4} b_{0 T}^{(+)}, \\
C_{T}^{\Delta s} & =-\frac{1}{2} b_{1 T}^{(-)}-\frac{1}{4} b_{1 T}^{(+)}, \\
C_{T}^{\tau} & =b_{0 T}^{(+)}, \\
C_{T}^{J} & =-b_{1 T}^{(+)} .
\end{aligned}
$$

In contrast to Eqs. A25-A28, Eqs. A30-A33 do not impose any constraints on the parameters $b_{S T}^{( \pm)}$, because the numbers of the independent parameters in the left and right sides of Eqs. A30 A33 are equal to each other. In this case Eqs. A29) are generally not fulfilled.

The definitions A30-A33) are convenient in the case when it is necessary to eliminate the so-called $J^{2}$ terms or/and the spin-spin terms from the residual interaction $V$. In most parametrizations of the Skyrme energy functional the $J^{2}$ terms are omitted by setting the constants $C_{T}^{J}$ to be equal to zero. To maintain self-consistency on the RPA level these constants should be equal to zero also in the residual interaction. In this case the parameters $b_{S T}^{( \pm)}$are determined by Eqs. A30 - A33 in which $C_{T}^{J}=0$ while the coupling constants $C_{T}^{\Delta \rho}, C_{T}^{\Delta s}$, and $C_{T}^{\tau}$ are determined by Eqs. (A34)-(A36) and (A25)-(A28). Note that in these definitions Eqs. (A25)- A28) play intermediate role (they do not give the final values of the parameters $b_{S T}^{( \pm)}$, so Eqs. A29 do not follow from them).

Sometimes in the RPA calculations of the excitations of the spherical even-even nuclei the spin-spin terms of $V$ are also omitted since this does not lead to the violation of the self-consistency. In the above equations it means that (i) the sum in Eq. A12 is restricted by the terms with $S=0$ and (ii) the constants $C_{T}^{\Delta s}$ in Eq. A33 are set to be equal to zero (the constants $C_{T}^{\tau}, C_{T}^{J}$, and $C_{T}^{\Delta \rho}$ are determined as described above).

\section{Spin-orbit term of the momentum-dependent part of the interaction}

$$
\begin{aligned}
& V_{(12,34)}^{J(2) S O}=\delta_{\tau_{1}, \tau_{2}} \delta_{\tau_{4}, \tau_{3}} W_{0}\left(1+x_{W} \delta_{\tau_{1}, \tau_{3}}\right) \\
& \times\left\{u_{(12,34)}^{J}+u_{(34,12)}^{J}\right. \\
& \left.+(-1)^{j_{1}-j_{2}+j_{3}-j_{4}}\left[u_{(21,43)}^{J}+u_{(43,21)}^{J}\right]\right\},
\end{aligned}
$$

where

$$
\begin{aligned}
& u_{(12,34)}^{J}=\frac{3}{4 \pi} \sum_{L=J, J \pm 1} \sum_{l_{1}^{\prime}=l_{1} \pm 1} \sum_{l_{3}^{\prime}=l_{3} \pm 1} a_{(12)}^{J L l_{1}^{\prime}} b_{(34)}^{J L l_{3}^{\prime}} \\
& \times \int_{0}^{\infty} d r r^{2} D_{(1)}^{l_{1}^{\prime}}(r) R_{(2)}(r) D_{(3)}^{l_{3}^{\prime}}(r) R_{(4)}(r),
\end{aligned}
$$

$$
\begin{aligned}
a_{(12)}^{J L l_{1}^{\prime}} & =\sum_{l_{1}^{\prime \prime}=j_{1} \pm \frac{1}{2}}(-1)^{l_{1}+l_{1}^{\prime \prime}}\left(2 l_{1}^{\prime}+1\right)\left(2 l_{1}^{\prime \prime}+1\right) \\
& \times \sqrt{\left(2 j_{1}+1\right)\left(2 l_{1}+1\right)\left(2 j_{2}+1\right)\left(2 l_{2}+1\right)} \\
& \times\left(\begin{array}{ccc}
l_{1} & l_{1}^{\prime} & 1 \\
0 & 0 & 0
\end{array}\right)\left(\begin{array}{ccc}
l_{2} & l_{1}^{\prime} & L \\
0 & 0 & 0
\end{array}\right) \\
& \times\left\{\begin{array}{ccc}
l_{1} & l_{1}^{\prime} & 1 \\
1 & 1 & l_{1}^{\prime \prime}
\end{array}\right\}\left\{\begin{array}{ccc}
l_{2} & l_{1}^{\prime} & L \\
1 & J & l_{1}^{\prime \prime}
\end{array}\right\} \\
& \times\left\{\begin{array}{ccc}
j_{1} & l_{1} & \frac{1}{2} \\
1 & \frac{1}{2} & l_{1}^{\prime \prime}
\end{array}\right\}\left\{\begin{array}{ccc}
j_{2} & l_{2} & \frac{1}{2} \\
l_{1}^{\prime \prime} & j_{1} & J
\end{array}\right\},
\end{aligned}
$$




$$
\begin{aligned}
& b_{(34)}^{J L l_{3}^{\prime}}=(-1)^{l_{3}+j_{3}-\frac{1}{2}}(2 L+1)\left(2 l_{3}^{\prime}+1\right) \\
& \times \sqrt{\left(2 j_{3}+1\right)\left(2 l_{3}+1\right)\left(2 j_{4}+1\right)\left(2 l_{4}+1\right)} \\
& \times\left(\begin{array}{ccc}
l_{3} & l_{3}^{\prime} & 1 \\
0 & 0 & 0
\end{array}\right)\left(\begin{array}{ccc}
l_{4} & l_{3}^{\prime} & L \\
0 & 0 & 0
\end{array}\right) \\
& \times\left\{\begin{array}{ccc}
j_{4} & l_{4} & \frac{1}{2} \\
l_{3} & j_{3} & J
\end{array}\right\}\left\{\begin{array}{ccc}
l_{4} & l_{3}^{\prime} & L \\
1 & J & l_{3}
\end{array}\right\}, \\
& D_{(1)}^{l_{1}^{\prime}}(r)=R_{(1)}^{\prime}(r)-\sqrt{6 l_{1}\left(l_{1}+1\right)\left(2 l_{1}+1\right)} \\
& \times\left\{\begin{array}{ccc}
1 & l_{1} & l_{1}^{\prime} \\
l_{1} & 1 & 1
\end{array}\right\} \frac{1}{r} R_{(1)}(r) .
\end{aligned}
$$

$W_{0}$ and $x_{W}$ in Eq. A38 are the parameters of the Skyrme energy functional (see [54]). Note that these parameters are related with the constants $b_{4}$ and $b_{4}^{\prime}$ of Ref. [49] by the formulas $W_{0}=2 b_{4}, x_{W}=b_{4}^{\prime} / b_{4}$.

\section{Coulomb term}

The Coulomb term has the non-zero matrix elements only for the proton single-particle wave functions. It con- sists of two parts

$$
V_{(12,34)}^{J(C)}=V_{(12,34)}^{J(C) D}+V_{(12,34)}^{J(C) X}
$$

where $V_{(12,34)}^{J(C) D}$ and $V_{(12,34)}^{J(C) X}$ are the direct and exchange terms, respectively.

For the direct term we have

$$
\begin{aligned}
V_{(12,34)}^{J(C) D} & =\frac{4 \pi e^{2}}{(2 J+1)^{2}} \\
& \times\left\langle j_{2} l_{2}\left\|T_{J J 0}\right\| j_{1} l_{1}\right\rangle\left\langle j_{4} l_{4}\left\|T_{J J 0}\right\| j_{3} l_{3}\right\rangle \\
& \times \int_{0}^{\infty} d r r^{2} \int_{0}^{\infty} d r^{\prime} r^{\prime 2} \frac{r_{<}^{J}}{r_{>}^{J+1}} \\
& \times R_{(1)}(r) R_{(2)}(r) R_{(3)}\left(r^{\prime}\right) R_{(4)}\left(r^{\prime}\right)
\end{aligned}
$$

where $r_{<}=\min \left(r, r^{\prime}\right), r_{>}=\max \left(r, r^{\prime}\right)$.

The exchange term is treated within the Slater approximation in consistency with the usual form of the Skyrme energy functional. In this approximation the expression for the exchange term is

$$
\begin{aligned}
V_{(12,34)}^{J(C) X} & =-\frac{(9 \pi)^{-1 / 3} e^{2}}{2 J+1} \\
& \times\left\langle j_{2} l_{2}\left\|T_{J J 0}\right\| j_{1} l_{1}\right\rangle\left\langle j_{4} l_{4}\left\|T_{J J 0}\right\| j_{3} l_{3}\right\rangle \\
& \times \int_{0}^{\infty} d r r^{2} \rho_{p}^{-2 / 3}(r) \\
& \times R_{(1)}(r) R_{(2)}(r) R_{(3)}(r) R_{(4)}(r)
\end{aligned}
$$

[1] S. Drożdż, S. Nishizaki, J. Speth, and J. Wambach, Phys. Rep. 197, 1 (1990)

[2] V. G. Soloviev, Theory of complex nuclei (Pergamon Press, Oxford, 1976)

[3] J. Dehesa, S. Krewald, J. Speth, and A. Faessler, Phys. Rev. C 15, 1858 (1977)

[4] V. I. Tselyaev, Yad.Fiz.; Soviet Journal of Nuclear Physics (English translation) 50, 1252 (1989)

[5] S. Kamerdzhiev, J. Speth, G. Tertychny, and V. Tselyaev, Nucl.Phys.A 555, 90 (1993), ISSN 0375-9474, http://www.sciencedirect.com/science/ article/pii/0375947493903150

[6] S. Kamerdzhiev, J. Speth, and G. Tertychny, Phys.Rep. 393, 1 (2004), arXiv:nucl-th/0311058 [nucl-th]

[7] N. Lyutorovich, V. I. Tselyaev, J. Speth, S. Krewald, F. Grümmer, and P. G. Reinhard, Phys. Rev. Lett. 109, 092502 (Aug 2012), http://link.aps .org/doi/10. 1103/PhysRevLett.109.092502

[8] N. Lyutorovich, V. Tselyaev, J. Speth, S. Krewald, F. Grümmer, and P.-G. Reinhard, Phys.Lett.B 749, 292 (2015)

[9] D. Vretenar, A. V. Afanasjev, G. Lalazissis, and P. Ring, Phys. Rep. 409, 101 (2005)

[10] M. Bender, P.-H. Heenen, and P.-G. Reinhard, Rev.Mod.Phys. 75, 121 (2003)

[11] S. Goriely, M. Samyn, P. H. Heenen, J. M. Pearson, and F. Tondeur, Phys. Rev. C 66, 024326 (Aug 2002), http:
//link.aps.org/doi/10.1103/PhysRevC.66.024326

[12] M. Kortelainen, T. Lesinski, J. Moré, W. Nazarewicz, J. Sarich, N. Schunck, M. V. Stoitsov, and S. Wild, Phys. Rev. C 82, 024313 (2010)

[13] J. Speth, E. Werner, and W. Wild, Phys. Rep. 33, 127 (1977)

[14] P.-G. Reinhard and Y. Gambhir, Ann. Phys. (Leipzig) 504, 598 (1992)

[15] P.-G. Reinhard, Ann. Phys. (Leipzig) 504, 632 (1992)

[16] J. P. Jeukenne, A. Lejeune, and C. Mahaux, Phys.Rept. 25, 83 (1976)

[17] A.B.Migdal, Theory of Finite Fermi Systems and Application to Atomic Nuclei (Wiley, New York, 1967)

[18] L. D. Landau, E. M. Lifshitz, and L. P. Pitajevski, Course of Theoretical Physics 9 - Statisical Physics (Pergamon press, Oxford, 1980)

[19] T. H. R. Skyrme, Nucl. Phys. 9, 615 (1959)

[20] J. W. Negele and D. Vautherin, Phys. Rev. C 5, 1472 (1972)

[21] D. Vautherin and D. Brink, Phys. Rev.C 5, 626 (1972)

[22] F. Grümmer and J. Speth, J.Phys.G:Nucl.Part.Phys. 32, R193 (2006)

[23] S. P. Kamerdzhiev, G. Y. Tertychny, and V. I. Tselyaev, Fiz. Elem. Chastits At. Yadra; Phys. Part. Nucl. 28, 333; 134 (1997)

[24] V. I. Tselyaev, Phys.Rev. C 75, 024306 (2007), arXiv:nucl-th/0505031 [nucl-th] 
[25] V. I. Tselyaev, Phys. Rev. C 88, 054301 (2013)

[26] J. R. Stone and P. . G. Reinhard, Prog. Part. Nucl. Phys. 58, 587 (2007), http://www.arxiv.org/abs/nuclth/0607002, http://dx.doi.org/10.1016/j.ppnp. 2006.07 .001

[27] J. Erler, P. Klüpfel, and P. G. Reinhard, J. Phys. G 38, 033101 (2011), doi:10.1088/0954-3899/38/3/033101

[28] P. Klüpfel, P. G. Reinhard, T. J. Bürvenich, and J. A. Maruhn, Phys. Rev. C 79, 034310 (Mar 2009), http: //link.aps.org/doi/10.1103/PhysRevC.79.034310

[29] M. Kortelainen, T. Lesinski, J. Moré, W. Nazarewicz, J. Sarich, et al., Phys. Rev. C 82, 024313 (2010), arXiv:1005.5145 [nucl-th]

[30] W. D. Myers, Droplet Model of Atomic Nuclei (IFI/Plenum, New York, 1977)

[31] J. Dobaczewski, W. Nazarewicz, and P.-G. Reinhard, J. Phys. G 41, 074001 (2014)

[32] J. Erler and P.-G. Reinhard, J. Phys. G 42, 034026 (2014)

[33] P.-G. Reinhard, Phys. Scr. 91, 023002 (2015), http:// dx.doi.org/10.1088/0031-8949/91/2/023002

[34] W. Nazarewicz, P. G. Reinhard, W. Satula, and D. Vretenar, Eur. Phys. J. A 50, 20 (2014), arXiv:1307.5782, http://dx.doi.org/10.1140/epja/i2014-14020-3

[35] J. Speth, S. Krewald, F. Grümmer, P. G. Reinhard, N. Lyutorovich, and V. Tselyaev, Nucl. Phys. A928, 17 (2014)

[36] P. Ring and P. Schuck, The Nuclear Many-Body Problem (Springer-Verl., New York, Heidelberg, Berlin, 1980)

[37] N. Lyutorovich, J. Speth, A. Avdeenkov, F. Grümmer, S. Kamerdzhiev, S. Krewald, and V. Tselyaev, Eur.Phys.J. A37, 381 (2008), arXiv:0806.2813 [nucl-th]

[38] A. Avdeenkov, F. Grümmer, S. Kamerdzhiev, S. Krewald, N. Lyutorovich, V. Tselyaev, and J. Speth, Bulletin of the Russian Academy of Sciences: Physics 73, 792 (2009), ISSN 1062-8738, http://dx.doi.org/10.3103/ S1062873809060203

[39] T. Sil, S. Shlomo, B. K. Agrawal, and P.G. Reinhard, Phys. Rev. C 73, 034316 (2006), http://www.arxiv.org/abs/nucl-th/0601091, //link.aps.org/doi/10.1103/PhysRevC.73.034316

[40] S. N. Belyaev, O. V. Vasiliev, V. V. Voronov, A. A. Nechkin, V. Y. Ponomarev, and V. A. Semenov, Phys.Atom.Nucl. 58, 1883 (1995)

[41] D. H. Youngblood, Y.-W. Lui, H. L. Clark, B. John, Y. Tokimoto, and X. Chen, Phys. Rev. C 69, 034315 (Mar 2004), http://link.aps.org/doi/10. 1103/PhysRevC.69.034315

[42] M. Brack, C. Guet, and H.-B. Håkansson, Phys. Rep. 123, 275 (1985)

[43] V. A. Erokhova, M. A. Elkin, A. V. Izotova, B. S. Ishkhanov, L. M. Kapitonov, E. I. Lileeva, and E. V. Shirokov, Izv.Ross.Akad.Nauk.Ser.Fiz.. 67, 1479 (2003)

[44] M. R. Anders, S. Shlomo, T. Sil, D. H. Youngblood, Y.-W. Lui, and Krishichayan, Phys. Rev. C 87, 024303 (Feb 2013), http://link.aps.org/doi/10. 1103/PhysRevC.87.024303

[45] C. Toepffer and P.-G. Reinhard, Ann. Phys. 181, 1 (1988)

[46] J. Erler, P. Klüpfel, and P. . G. Reinhard, J. Phys. G 37, 064001 (2010), http://www.arxiv.org/abs/1002.0027, http://dx.doi.org/10.1088/0954-3899/37/6/064001

[47] Y. M. Engel, D. M. Brink, K. Goeke, S. J. Krieger, and D. Vautherin, Nucl. Phys. A 249, 215 (1975)

[48] S. K. Bogner, R. Furnstahl, and A. Schwenk, Prog. Part. Nucl. Phys. 65, 94 (2010)

[49] P. G. Reinhard and H. Flocard, Nucl. Phys. A 584, 467 (1995)

[50] D. J. Thouless, The Quantum Mechanics of Many-Body Systems (Academic Press, New York, 1961)

[51] P.-G. Reinhard, M. Bender, W. Nazarewicz, and T. Vertse, Phys. Rev. C 73, 014309 (2006)

[52] E. Chabanat, P. Bonnche, P. Haensel, J. Meyer, and R. Schaefer, Nucl. Phys. A635, 231 (1998)

[53] E. V. Litvinova and V. I. Tselyaev, Phys. Rev. C 75, 054318 (2007)

[54] M. M. Sharma, G. Lalazissis, J. König, and P. Ring, Phys. Rev. Lett. 74, 3744 (1995) 